\title{
El uso del fuego en el complejo arqueológico de las huacas del Sol y de la Luna, Perú. Un primer ensayo de tipología de las áreas de combustión
}

L'utilisation du feu dans le complexe archéologique de huacas del Sol y de la Luna, Pérou. Un essai de typologie des zones de combustion

The use of fire at the archaeological complex of huacas del Sol y de la Luna, Peru. An initial typology of combustion areas

Feren Castillo Luján, Santiago Uceda Castillo y Ramiro Javier March

\section{OpenEdition}

Edición electrónica

URL: http://journals.openedition.org/bifea/7425

DOI: $10.4000 /$ bifea.7425

ISSN: 2076-5827

Editor

Institut Français d'Études Andines

Edición impresa

Fecha de publicación: 1 abril 2015

Paginación: 53-59

ISSN: 0303-7495

Referencia electrónica

Feren Castillo Luján, Santiago Uceda Castillo y Ramiro Javier March, « El uso del fuego en el complejo arqueológico de las huacas del Sol y de la Luna, Perú. Un primer ensayo de tipología de las áreas de combustión », Bulletin de l'Institut français d'études andines [En línea], 44 (1) | 2015, Publicado el 08 mayo 2015, consultado el 05 noviembre 2020. URL : http://journals.openedition.org/bifea/7425; DOI : https://doi.org/10.4000/bifea.7425

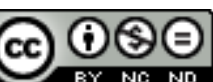

Les contenus du Bulletin de l'Institut français d'études andines sont mis à disposition selon les termes de la licence Creative Commons Attribution - Pas d'Utilisation Commerciale - Pas de Modification 4.0 International. 


\title{
El uso del fuego en el complejo arqueológico de las huacas del Sol y de la Luna, Perú. Un primer ensayo de tipología de las áreas de combustión
}

\author{
Feren Castillo Luján* \\ Santiago Uceda Castillo** \\ Ramiro Javier March ${ }^{* * *}$
}

\begin{abstract}
Resumen
Poca atención se ha brindado al estudio de las estructuras de combustión en los sitios urbanos del área andina central. Nuestro estudio se ha centrado en una muestra de las áreas de combustión registradas en casi 20 años de estudio en el complejo arqueológico huacas de Sol y de la Luna. Este estudio se ha iniciado con una propuesta de clasificación tipológica de dichas estructuras, así como una primera propuesta de su funcionalidad a partir del estudio contextual arquitectónico, los estudios etnohistóricos y etnográficos. Futuros análisis químicos y físicos, tanto de las estructuras como de los sedimentos asociados, así como ensayos experimentales, corroborarán o no esta primera propuesta.
\end{abstract}

Palabras clave: áreas de combustión, tipología, cultura moche, huacas del Sol y de la Luna

\section{L'utilisation du feu dans le complexe archéologique de huacas del Sol y de la Luna, Pérou. Un essai de typologie des zones de combustion}

\section{Résumé}

L'étude des structures de combustion dans les sites urbains dans la région centrale des Andes est peu developpée jusqu'à présent. Notre étude a porté sur un échantillon des zones de combustion

* Universidad Nacional de Trujillo, Proyecto Arqueológico Huacas del Sol y de la Luna. E-mail: ferencastillo@hotmail.com

** Universidad Nacional de Trujillo, Proyecto Arqueológico Huacas del Sol y de la Luna. E-mail: santiago_uceda@hotmail.com

${ }^{* * *}$ CR1 CNRS, CREAAH UMR 6566 du CNRS Centre de recherches en Archéologie, Archéosciences et Histoire. E-mail: ramiro.march@univ-rennes1.fr 
enregistrées durant près de 20 ans dans le complexe archéologique huacas del Sol y de la Luna. Cet article constitue une première classification typologique de ces structures, ainsi qu'une première approche de leurs fonctionnalités à partir des études contextuelles architecturales, ethnohistoriques et ethnographiques. Dans le futur, des analyses chimiques et physiques, ainsi que des études sur les structures architecturales, des études sédimentologiques, et des essais expérimentaux confirmeront ou non cette première proposition.

Mots-clés: zones de combustion, typologie, civilisation Mochica, huacas del Sol y de la Luna

\title{
The use of fire at the archaeological complex of huacas del Sol y de la Luna, Peru. An initial typology of combustion areas
}

\begin{abstract}
Little attention has been given to the study of combustion structures in the Prehispanic urban sites of the Central Andes. Our study focused on a sample of the combustion areas registered during the 20 years of research at Huacas del Sol y de la Luna. This study begins with a proposed typology for these structures, as well as an initial proposal concerning their functionality based an analysis of their architectural context, as well as ethnohistoric and ethnographic studies. Future chemical and physical analyses of the structures as well as the associated sediments, complemented by experimental tests will permit the evaluation of our initial proposal..
\end{abstract}

Keywords: combustion areas, typology, Moche culture, huacas del Sol y de la Luna

\section{INTRODUCCIÓN}

En los estudios del urbanismo andino (500 a. C. - 1532 d. C.) se ha prestado poca atención a la información sobre el rol del fuego, tanto en la utilización doméstica como simbólica o artesanal. Sin embargo, este podría brindar información sobre los grupos sociales estudiados y la naturaleza de las ocupaciones mochicas en la zona urbana de las huacas del Sol y de la Luna, ya sea desde un punto de vista de su organización arquitectónica, funcional o estructural. Como es sabido, la utilización del fuego está estrechamente ligada a nuestra evolución desde un punto de vista biológico o cultural. El fuego tiene también un aspecto integrador en las relaciones sociales ya que el hombre se socializa a través de su utilización, la cual es compartida frecuentemente (Leroi-Gourhan, 1964; Levi-Strauss, 1964; Lieberherr, 2006; March, 2002; Wrhangham, 2009).

Como fuente de energía ha permitido transformar la materia prima o la cocción de alimentos y bebidas alcoholizadas. Este también ha sido utilizado en la preparación de variados colores del ocre mineral en bruto que fue utilizado para pintar el cuerpo, la cerámica o las fachadas y pisos de los templos. Ha servido también para preparar los terrenos para las siembras y ha jugado sin duda un rol selectivo en cuanto a la explotación de plantas desde el descubrimiento de la agricultura. Finalmente, el fuego ha sido útil en la organización de actividades, así como en la integración del grupo al interior y exterior del hábitat contribuyendo a la domesticación del espacio y su organización (March, 2002), y ha determinado en cierta forma la organización arquitectónica del mismo (Gousdblom, 1992; Wright, 
1966). Esta estructuración del espacio no responde solamente a las imposiciones técnicas de la utilización del fuego en el hogar sino que refleja la estructuración social de cada grupo humano (Bourdieu, 1980).

El estudio de la utilización del fuego por parte de los grupos que habitaron las huacas del Sol y de la Luna puede darnos una valiosa información sobre los cambios sociales acontecidos dentro de los procesos históricos que previamente condujeron al pasaje de un estado teocrático a un estado secular (Uceda, 2010). Para ello, este artículo propone una primera propuesta tipológica a partir de la clasificación de las diversas áreas de combustión, teniendo en cuenta la forma y función de estos elementos hallados en el sitio.

\section{METODOLOGÍA DE INVESTIGACIÓN}

Estudiar las áreas de combustión en el complejo arqueológico huacas del Sol y de la Luna implica una serie de problemas metodológicos a resolver, puesto que estas han sido elaboradas inicialmente para estudiar sociedades menos complejas (Leroi-Gourhan \& Brézillon, 1972; Olive \& Taborin, 1989; Bar-Yosef et al., 1996; Frère Sautor et al., 2003; March \& Wunsch, 2003). La estructura social diferenciada en este sitio moche posee una mayor densidad de población que produce bienes ${ }^{1}$ y alimentos, con un control de la energía térmica mucho más sofisticado y que se aplica en relación con la calidad y cantidad de los productos necesarios para el funcionamiento del aparato estatal. Por ello, fue necesario adecuar el concepto de área de combustión para referirnos a los diversos espacios físicos en el que se ha desarrollado una combustión o donde se han documentado restos asociados a la misma (Soler, 2003). La realización de un inventario de las áreas de combustión requiere de una tipología previa que, como se sabe, no representará el estado final de la investigación sobre la historia que condujo a los tipos definidos. Esta tipología podrá ser modificada en función de los resultados analíticos y experimentales que se realizarán en una segunda etapa de este estudio.

La investigación consistió en revisar el centenar de áreas de combustión registradas en diversos niveles de ocupación a lo largo de más de 15 años de investigación en el sitio (Castillo, 2012). De esta manera se propuso una clasificación en función de las características formales, como orientación, dimensiones, superficie de uso, contenido interno y externo, y la ubicación de cada estructura al interior de cada ambiente. Posteriormente se realizó el análisis y comparación con la iconografía

1 Los estados arcaicos están íntimamente relacionados con la presencia de centros urbanos, muchas veces del tipo ciudad. En las ciudades, una de las características fundamentales es la presencia de especialistas que requieren espacios y estructuras arquitectónicas específicas donde desarrollar sus actividades productivas. En este sentido, la presencia de estos artesanos especializados requiere además de estructuras de combustión muy variadas; por lo que esperamos encontrar en el registro arqueológico una mayor diversidad de estas estructuras, de acuerdo a las actividades de cada especialista. Es decir, esperamos que las estructuras de combustión para un ceramista que hace cocción reductora sean diferentes de aquel que hace una cocción oxidante; lo mismo debe ser para un metalurgista o un orfebre. 
moche, los datos etnohistóricos y etnográficos de viviendas rurales, y los estudios e hipótesis que han sido construidas previamente sobre estas estructuras por otros investigadores del mundo andino, interesados en el estudio de la energía térmica y su aplicación. De esta manera, nuestra muestra corresponde a diez conjuntos arquitectónicos del núcleo urbano; así como dos paramentos con evidencias de incendio del templo viejo de huaca de la Luna (fig. 1).

El Conjunto Arquitectónico 5 (CA5) fue excavado como muestra de análisis y se ubica al sur del núcleo urbano Moche (figs. 1 y 2). El resultado de la excavación permitió definir un nuevo subconjunto (CA5-SC5) y un área no definida para la ocupación del piso 2 (Meneses et al., 2012). El CA5-SC5 se caracteriza por ser un espacio para celebraciones y servicios. La muestra empleada está representada por fogones ( $\mathrm{fg}$ ) y concentraciones de ceniza (cc) con siete casos respectivamente, distribuidos en ocho ambientes: 5-13 (cc3 y cc4), 5-19 (fg14), 5-22 (fg9, fg10 y fg17), 5-26 (cc6), 5-28 (cc10), 5-30 (fg12 y cc8), 5-35 (fg11 y cc9) y 4-5 (fg16 y cc7). En el área no definida se emplearon los elementos registrados en los ambientes 5-6 (fg19, fg20 y cc2) y 5-7 (fg15).

Los Conjuntos Arquitectónicos 7 y 9 se ubican en el centro del núcleo urbano (figs. 1 y 3) y han sido excavados hasta definir el piso de la última ocupación — piso 1- (Chapdelaine, 1998; Armas et al., 2007). El primer conjunto -a pesar de no haber sido definido completamente - fue incluido en la muestra por presentar un horno orfebre (Ho1). Se trata de un caso único ubicado al interior del ambiente 7-14, que además sugieren su función como taller orfebre. Además se han registrado tres concentraciones de cenizas en los ambientes 7-5b (cc1), 7-8 (cc2) y 7-10 (cc3). El conjunto 9 fue definido en su totalidad y está compuesto por áreas de cocina, molienda, depósitos, vestíbulos, etc. La muestra está representada por siete fogones distribuidos en los ambientes 9-10 (fg1), 9-12 (fg2), 9-13 (fg3), 9-28 (fg4), 9-35 (fg5 y fg6) y 9-25b (fg7).

Al oeste de la avenida 1, en el sector central del núcleo urbano se han definido dos bloques arquitectónicos para la ocupación del piso 2 (figs. 1 y 4). El primer bloque - al sur - está conformado por los Conjuntos Arquitectónicos 27 y 30 (Chiguala et al., 2012), que a su vez se subdividen en cinco áreas de actividad complementarias (Uceda, 2010). En el área administrativa se han registrado cuatro fogones distribuidos en los ambientes 27-34 (fg11), 27-35 (fg3), 27-39 (fg4 y fg5). En el área de servicios se ha definido un solo fogón de grandes dimensiones al interior del ambiente 27-9 (fg6). En el taller orfebre se registró la presencia de hornos al interior del ambiente 27-30 (Ho1, Ho2 y Ho3) y otros contextos como yunques y desechos de fundición. El área residencial se caracteriza por presentar un patio con banquetas y cuatro cocinas, donde se han registrado fogones y concentraciones de ceniza distribuidos en los ambientes 30-34a/c/d (fg7a), 27-32 (fg8), 30-17 (cc1), 30-35 (cc2), 30-38 (fg7b), 30-20 (fg9a, fg9b y cc3) y 30-2a/b (fg8a y fg8b). El área residencial y de servicios presenta dos fogones al interior del ambiente 30-22 (fg4 y fg5).

El segundo bloque está conformado por los Conjuntos Arquitectónicos 35, 17 y 21 (Chiguala et al., 2007), a su vez se subdivide en seis áreas (Uceda, 2010). En 


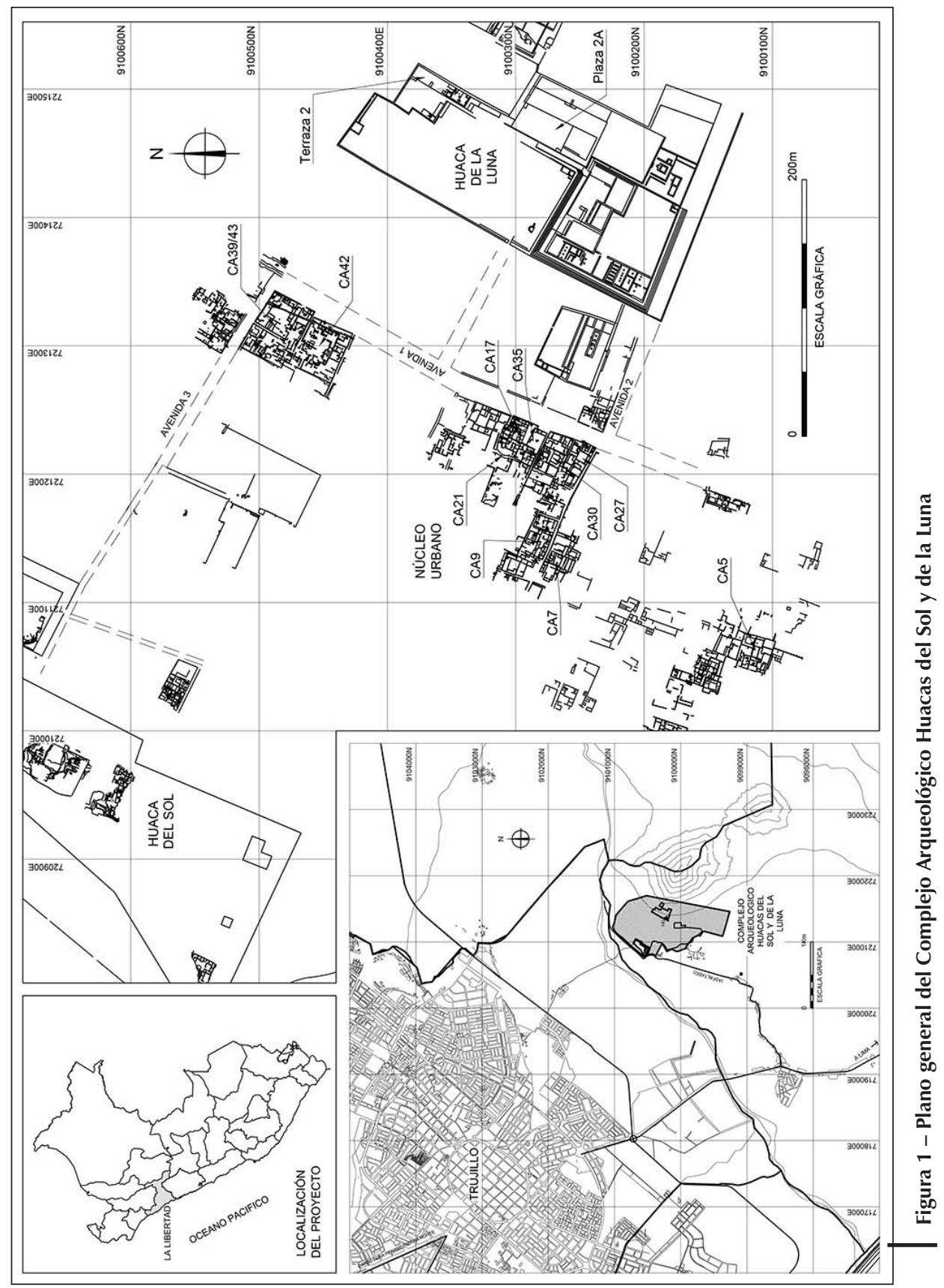


Feren Castillo Luján, Santiago Uceda Castillo, Ramiro Javier March

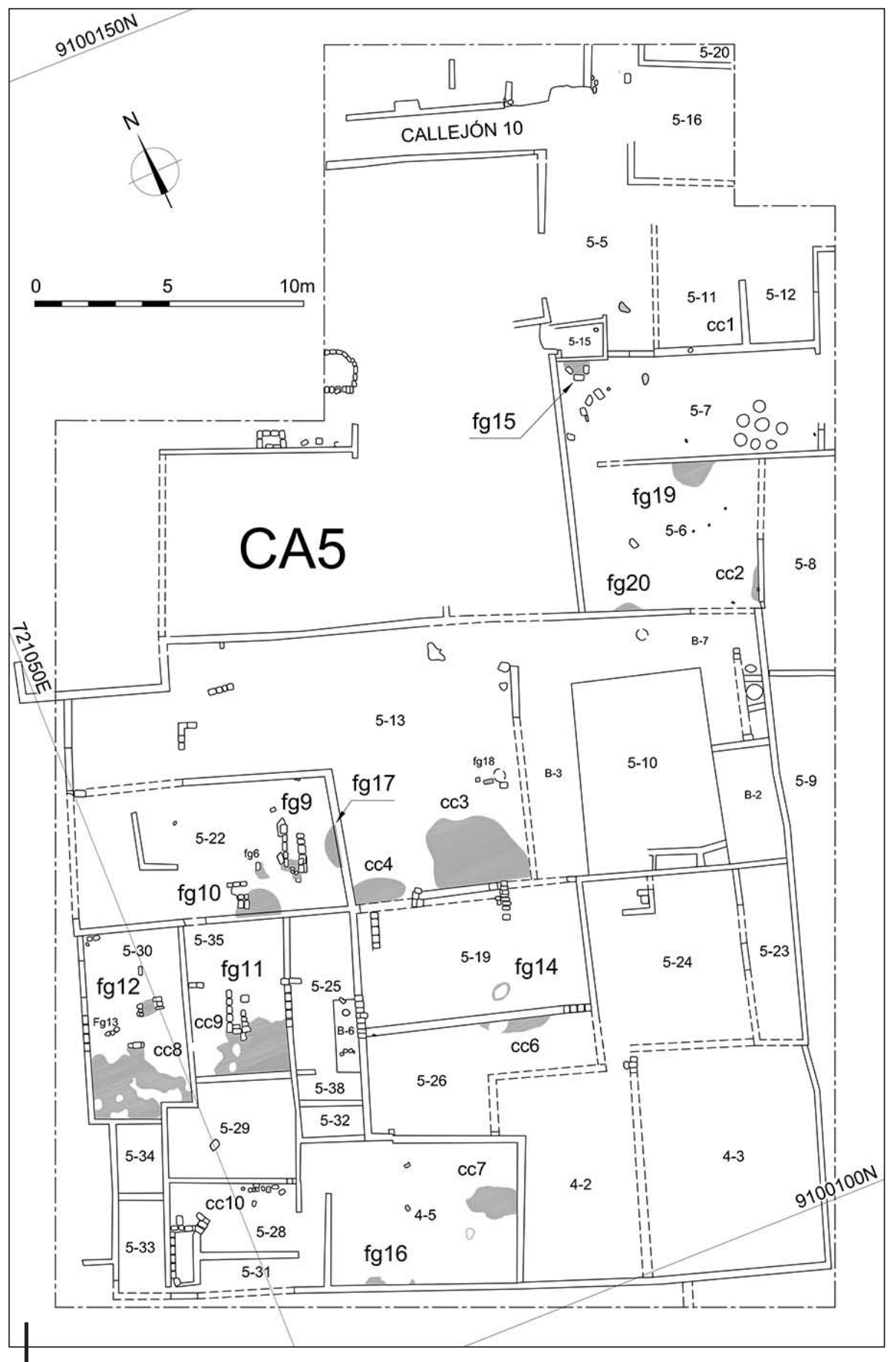

Figura 2 - Plano del Conjunto Arquitectónico 5 (CA5) 
El uso del fuego en el complejo arqueológico de las huacas del Sol y de la Luna, Perú

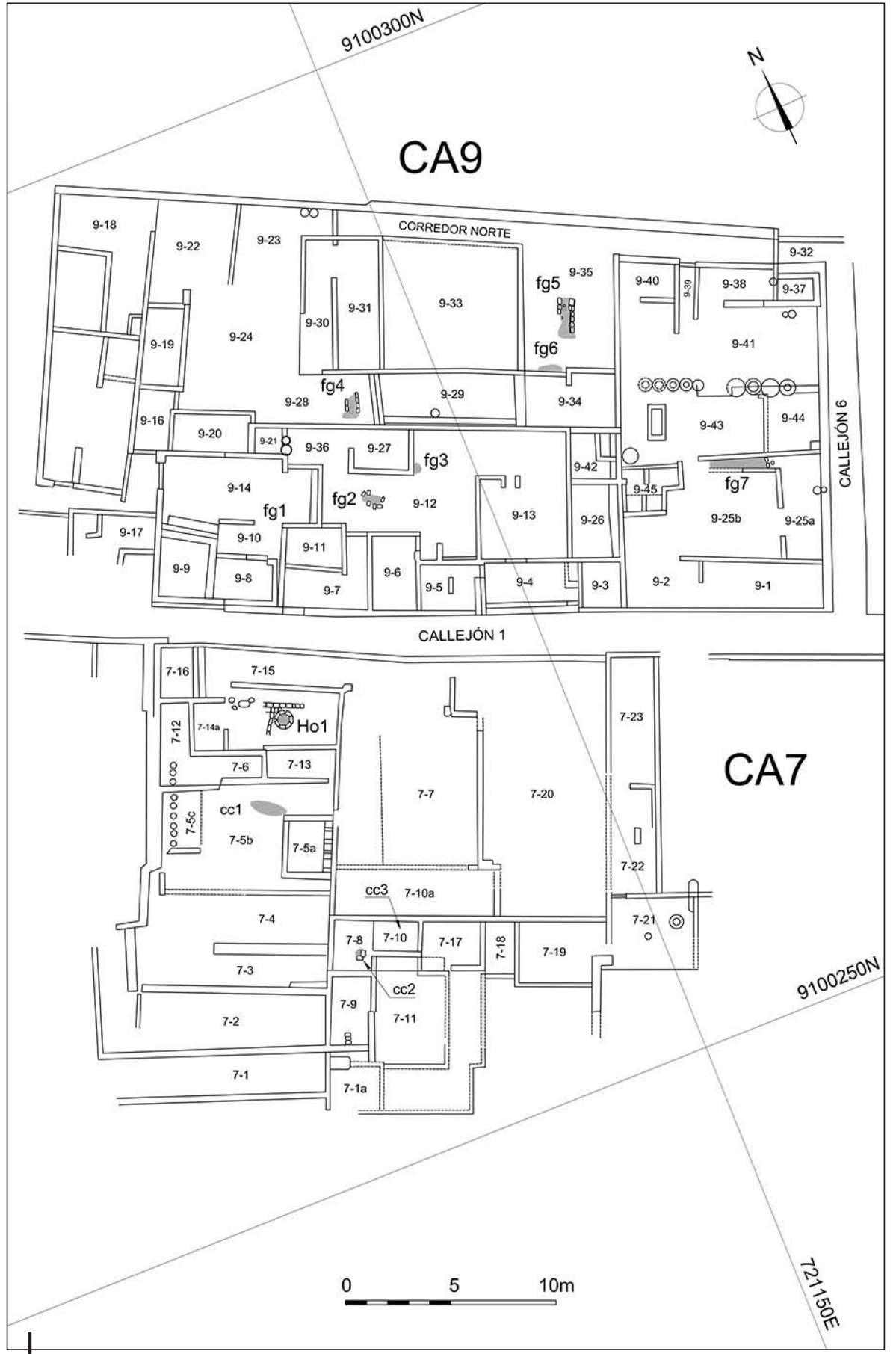

Figura 3 - Plano de los Conjuntos Arquitectónicos 7 y 9 (CA7 y CA9) 
Feren Castillo Luján, Santiago Uceda Castillo, Ramiro Javier March

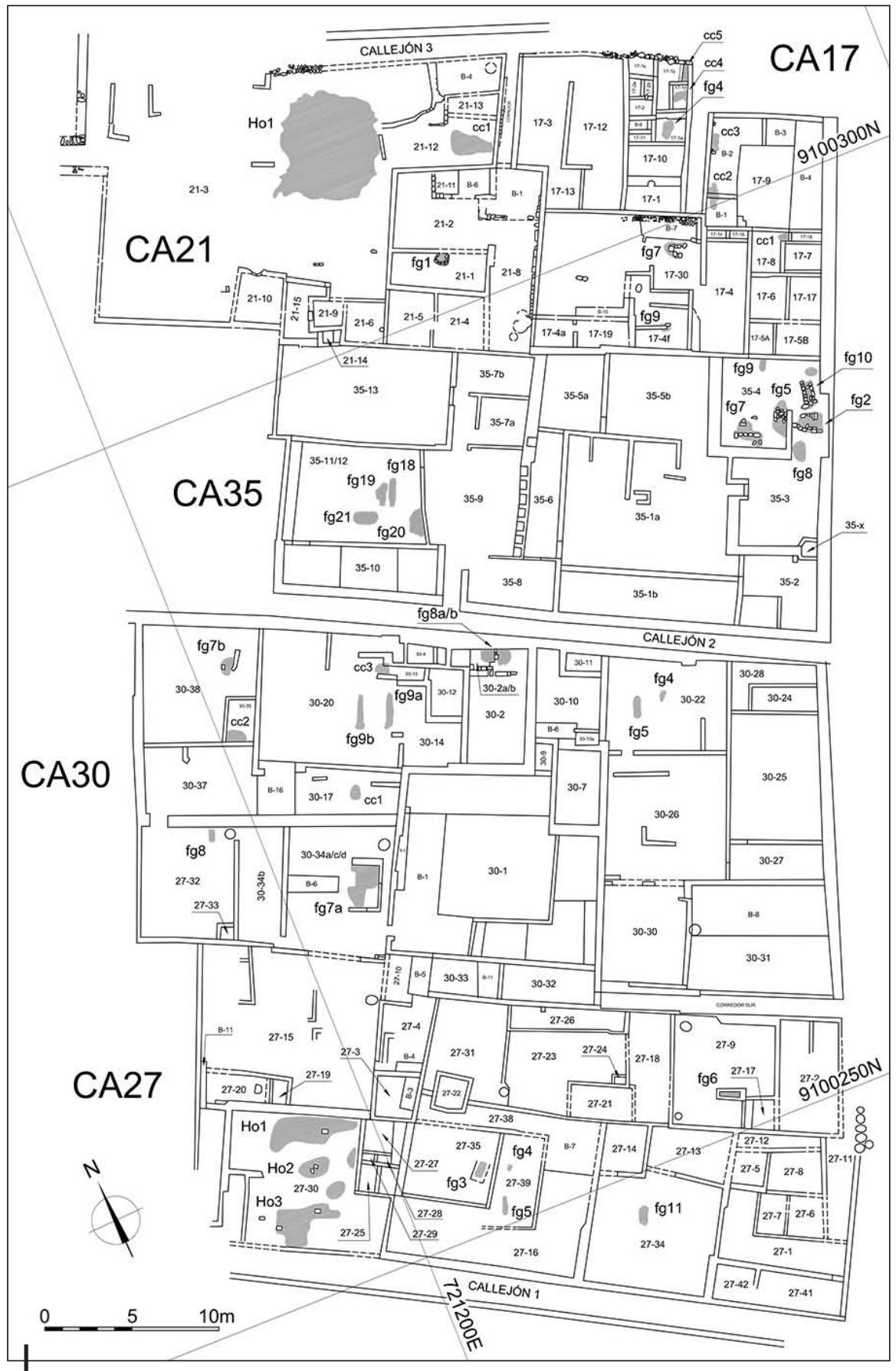

Figura 4 - Plano de los Bloques Arquitectónicos 1 (CA27 y CA30) y 2 (CA35, CA17 y CA21) 
el área de residencia y ritual se han registrado seis fogones en el ambiente 35-4 (fg2, fg5, fg7, fg8, fg9 y fg10), mientras en el área de servicios se definieron cuatro fogones al interior del ambiente 35-11/12 (fg18, fg19, fg20 y fg21). En el taller de abalorios de piedra se han registrado un fogón en el ambiente 17-10 (fg4) y dos concentraciones de ceniza en los ambientes 17-1b y 17-1d. En cambio, en el área de residencia o viviendas se han registrado dos fogones en los ambientes 17-30 (fg7) y 17-4f (fg9), además de tres concentraciones de cenizas registradas en los ambientes 17-8 y 17-9. En el área administrativa se ha definido un fogón en el ambiente 21-1; mientras en el taller alfarero se definieron un horno (Ho1) al interior del ambiente 21-3 y una concentración de ceniza (ambiente 21-2).

En el sector norte del núcleo urbano, también se han definido dos bloques arquitectónicos (figs. 1 y 5), cuyas formas arquitectónicas son muy similares y se les asocia a celebraciones y servicios (Seoane et al., 2010; Gayoso, 2010). El bloque 3 está conformado por el Conjunto Arquitectónico 42 para la ocupación del piso 2. Para esta ocupación se han registrado cuatro concentraciones de ceniza en los ambientes 42-3, 42-5 y 42-16; asimismo se han definido seis fogones distribuidos en los ambientes 42-6 (fg15), 42-3 (fg16), 42-4b (fg17), 42-13 (fg18), 42-17 (fg19) y 42-40 (fg20).

El bloque 4 está conformado por el Conjunto Arquitectónico 39/43 para la ocupación del piso 3 (Meneses et al., 2011). Para esta ocupación se han definido once concentraciones de ceniza y catorce fogones distribuidos en los ambientes 39-6E (fg18, cc1, сc2 у сс3), 39-7 (fg12), 39-8 (fg8), 39-11 (fg15), 39-16 (fg19), 39-19 (cc4), 39-20 (fg16, fg17 у cc5), 39-27 (fg20, fg21 у cc6), 43-5b (cc7), 43-10 (fg22), 43-16 (cc8), 43-23 (fg23 y fg24), 43-31 (fg25), 43-32 (fg26), 43-44 (cc9), 43-52 (cc10 y cc1).

Finalmente, en la muestra se han incluido dos casos únicos — hasta la fecha - de paramentos con evidencias de incendio, asociados a espacios ceremoniales del último edificio del Templo Viejo de huaca de la Luna. Ambos casos fueron registrados en los paramentos internos de los muros perimétricos este, tanto de la terraza 2 de Plaza 1 (Orbegoso et al., 2011), como de la plaza 2A (Chávarri \& Mejía, 2011).

\section{TIPOLOGÍA}

Las áreas de combustión analizadas corresponden a un total de 107 casos inmuebles (cuadro 1), divididos en tres clases: estructuras de combustión que hace referencia a la combustión in situ $(65,4 \%$ del total), concentraciones de ceniza $(32,7 \%)$ y testigos de incendio $(1,9 \%)$.

\section{1. Las estructuras de combustión}

El concepto estructuras de combustión nace con el resultado de las investigaciones en el sitio de Pincevent (Leroi-Gourhan \& Brézillon, 1972). El uso de este concepto permite abocar de manera general a las diferentes formas distribuidas y 
Feren Castillo Luján, Santiago Uceda Castillo, Ramiro Javier March

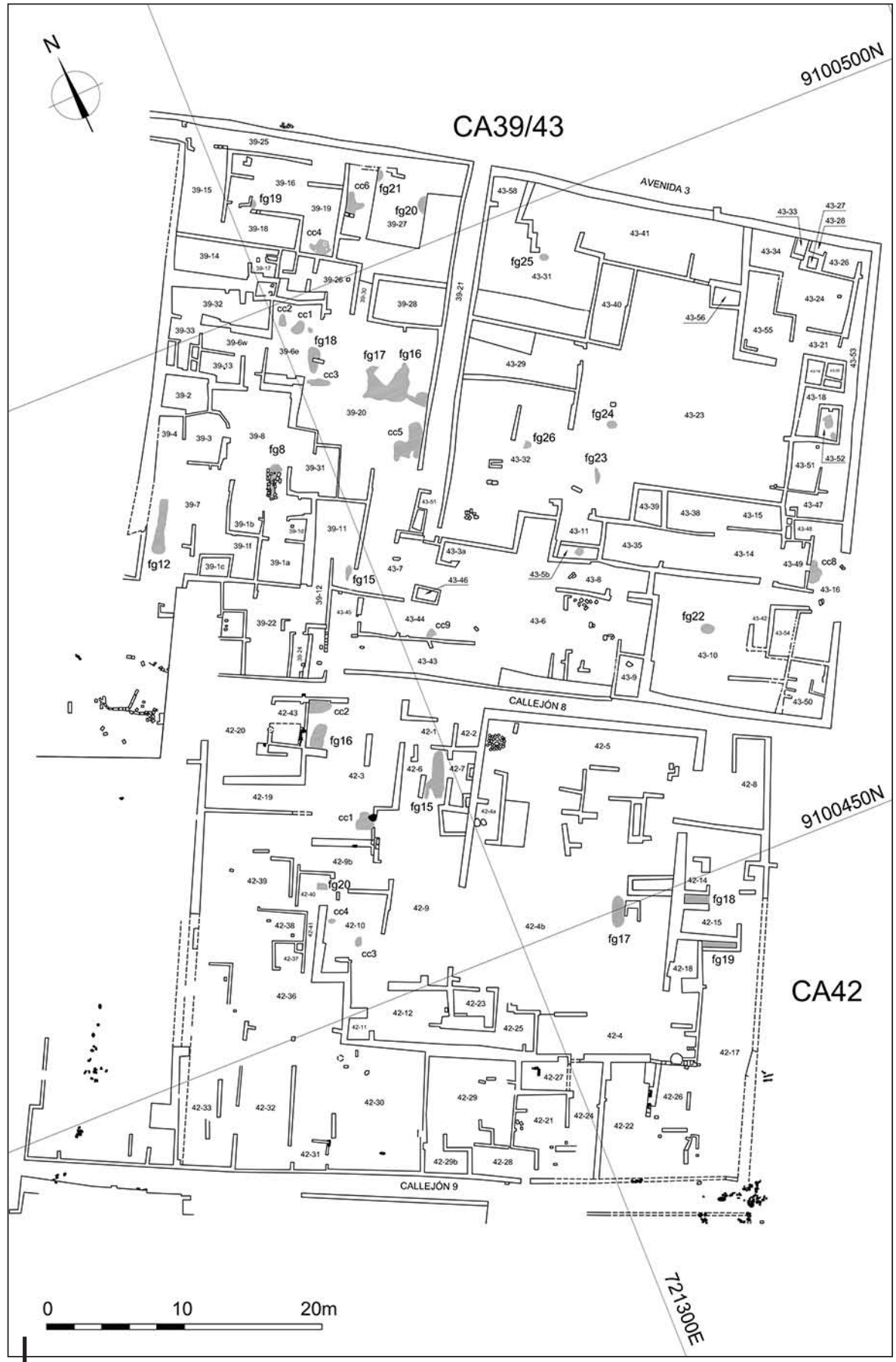

Figura 5 - Plano de los Bloques Arquitectónicos 3 (CA42) y 4 (CA39/43) 
El uso del fuego en el complejo arqueológico de las huacas del Sol y de la Luna, Perú

Cuadro 1 - Distribución de los casos de estructuras de combustión (fogones, hornos y brasero), concentraciones de ceniza y testigos de incendio

\begin{tabular}{|c|c|c|c|c|c|}
\hline Clase & Grupo & Tipo & Subtipo & $\#$ & Casos \\
\hline \multirow{14}{*}{ 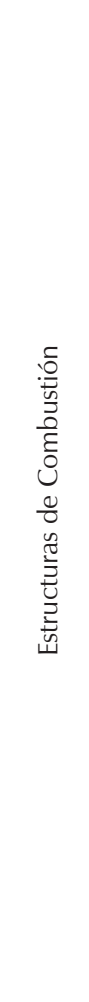 } & \multirow{9}{*}{ Fogón } & \multirow{4}{*}{ Fosa } & Simple & 21 & $\begin{array}{l}\text { CA17 (fg4a y fg9), CA27 (fg4, fg5 y fg11), CA30 } \\
\text { (fg4, fg5, fg8, fg9a y fg9b), CA35 (fg8, fg9, fg18, } \\
\text { fg19 y fg21), CA39/43 (fg15 y fg23) y CA42 (fg16, } \\
\text { fg17, fg18 y fg20) }\end{array}$ \\
\hline & & & $\begin{array}{l}\text { De paredes } \\
\text { verticales }\end{array}$ & 5 & CA39/43 (fg12, fg16, fg17, fg18 y fg19) \\
\hline & & & Simple con borde & 8 & $\begin{array}{l}\text { CA5 (fg9, fg11 y fg12), CA9 (fg1 y fg2), CA27 (fg3), } \\
\text { CA30 (fg7b) y CA35 (fg10) }\end{array}$ \\
\hline & & & $\begin{array}{l}\text { De paredes } \\
\text { verticales y borde }\end{array}$ & 7 & $\begin{array}{l}\text { CA9 (fg4 y fg5), CA35 (fg2, fg5 y fg7), CA39/43 } \\
\text { (fg8) y CA42 (fg15) }\end{array}$ \\
\hline & & \multicolumn{2}{|c|}{ De estructura rectangular } & 4 & CA9 (fg7), CA27 (fg6), CA30 (fg7a) y CA42 (fg19) \\
\hline & & \multirow[t]{2}{*}{$\begin{array}{l}\text { Adosado } \\
\text { al muro }\end{array}$} & Sin borde & 12 & $\begin{array}{l}\text { CA5 (fg10, fg16, fg17, fg19 y fg20), CA9 (fg3 y } \\
\text { fg6), CA30 (fg8a y fg8b), CA35 (fg20) y CA39/43 } \\
\text { (fg20 y fg21) }\end{array}$ \\
\hline & & & Con borde & 2 & CA5 (fg15) y CA21 (fg1) \\
\hline & & \multicolumn{2}{|l|}{ Plano } & 5 & CA5 (fg14) y CA39/43 (fg22, fg24, fg25 y fg26) \\
\hline & & \multicolumn{2}{|l|}{ Atípico } & 1 & CA17 (fg7) \\
\hline & \multirow{4}{*}{ Horno } & Alfarero & Abierto & 1 & CA21 (Ho1) \\
\hline & & \multirow{3}{*}{ Orfebre } & $\begin{array}{l}\text { De fusión con } \\
\text { chimenea }\end{array}$ & 1 & CA7 (Ho1) \\
\hline & & & De fusión cerrado & 1 & $\mathrm{CA} 27(\mathrm{Ho} 1)$ \\
\hline & & & De recocido & 2 & CA27 (Ho2 y Ho3) \\
\hline & \multicolumn{3}{|l|}{ Brasero } & - & Iconografía del mural de Pañamarca \\
\hline \multicolumn{4}{|c|}{ Concentraciones de ceniza } & 35 & 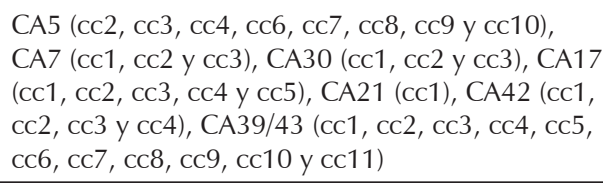 \\
\hline \multicolumn{4}{|c|}{ Testigo de incendio } & 2 & $\begin{array}{l}\text { MPE de plaza } 2 \text { A y MPE de terraza } 2 \text { de huaca de } \\
\text { la Luna }\end{array}$ \\
\hline
\end{tabular}

ordenadas particularmente por el hombre y que han sido alteradas por efecto de la combustión.

A partir de otras investigaciones (Arteaga et al., 2001) se sugieren dos rasgos importantes que permiten identificarlas: (1) Alteración térmica del sustrato sedimentario delimitado y uniforme, marcado por una variación cromática y por el grado de compactación del sedimento; (2) Presencia de residuos de combustión, como cenizas, carbones y material cultural alterados térmicamente dispuestos en un grado de orden latente o evidente. 
Las estructuras de combustión son las más comunes y se dividen en tres grupos: fogones (65 casos), hornos (5 casos) y braseros, que a su vez nos permiten proponer ocho tipos (fig. 6). Los braseros corresponden a un tipo de fuego portátil o mueble que podría estar destinado a funciones diferentes según los casos; por estas razones no se los incluye en los análisis estadísticos.

\section{1. 1. Los Fogones}

Los fogones son el grupo de estructuras más común (fig. 7). Se trata de un fuego abierto estructurado y construido en un espacio diferenciado de la vivienda, llamado cocina. La cocina, como espacio contenedor, puede estar parcial o totalmente techada, con el fin de generar la ventilación y fluido del aire. Todos los casos presentaban relleno de cenizas y carbones, con fragmentos de cerámica, lítico y restos orgánicos.

Haciendo una división en nueve lados del ambiente contenedor (fig. 8), notamos que los fogones se distribuyen recurrentemente en la parte central $(26,2 \%)$, seguido por el centro-este $(24,6 \%)$, norcentral $(13,8 \%)$, suroeste $(10,8 \%)$, surcentral $(9,2 \%)$ y centro-oeste $(4,6 \%)$. Además, el $63,1 \%$ del total de la muestra no está adosado a un muro, mientras el 36,9\% restante sí (fig. 9). Es notable, también, que el $67,7 \%$ de los casos están orientados de sur a norte, mientras el 32,3\% restante está orientado de este a oeste (fig. 10).

Los fogones, a partir de su forma arquitectónica y corte de perfil, se pueden dividir en: fogón tipo fosa (tipo A), fogón de estructura rectangular (tipo B), fogón adosado al muro (tipo C), fogón tipo plano (tipo D) y fogón atípico o diverso (tipo E).

\section{Tipo A: fogón tipo fosa}

Este tipo es el más común de la muestra estudiada, está representado con 41 casos, equivalente al 58,6\% del total de estructuras de combustión. Se han identificado 5 subtipos a partir de sus formas arquitectónicas más resaltantes: (A1) fogón tipo fosa simple, (A2) fogón tipo fosa de paredes verticales, (A3) fogón tipo fosa simple con borde, (A4) fogón tipo fosa de paredes verticales con borde.

\section{Subtipo A1: fogón tipo fosa simple}

Este subtipo generalmente está ubicado en el centro del ambiente contenedor. Presenta una planta variable de forma elíptica, ovoide o rectangular con bordes cortados. Vista de perfil se trata de una fosa cavada en el piso de forma cóncava, cuya profundidad varía entre los 0,05 y 0,10 m. Con respecto al eje mayor está orientado de sur a norte en el $85 \%$ de los casos, con algunas variables menores de este a oeste (fig. 6 y 11).

En la muestra se han identificado 21 casos, equivalentes al 30\% (fig. 7; ver cuadro 1). En todos los casos el borde presenta alteración térmica, producto de la oxidación de los sedimentos por acción del calor, mientras la fosa presenta ceniza adherida a las paredes. 
El uso del fuego en el complejo arqueológico de las huacas del Sol y de la Luna, Perú

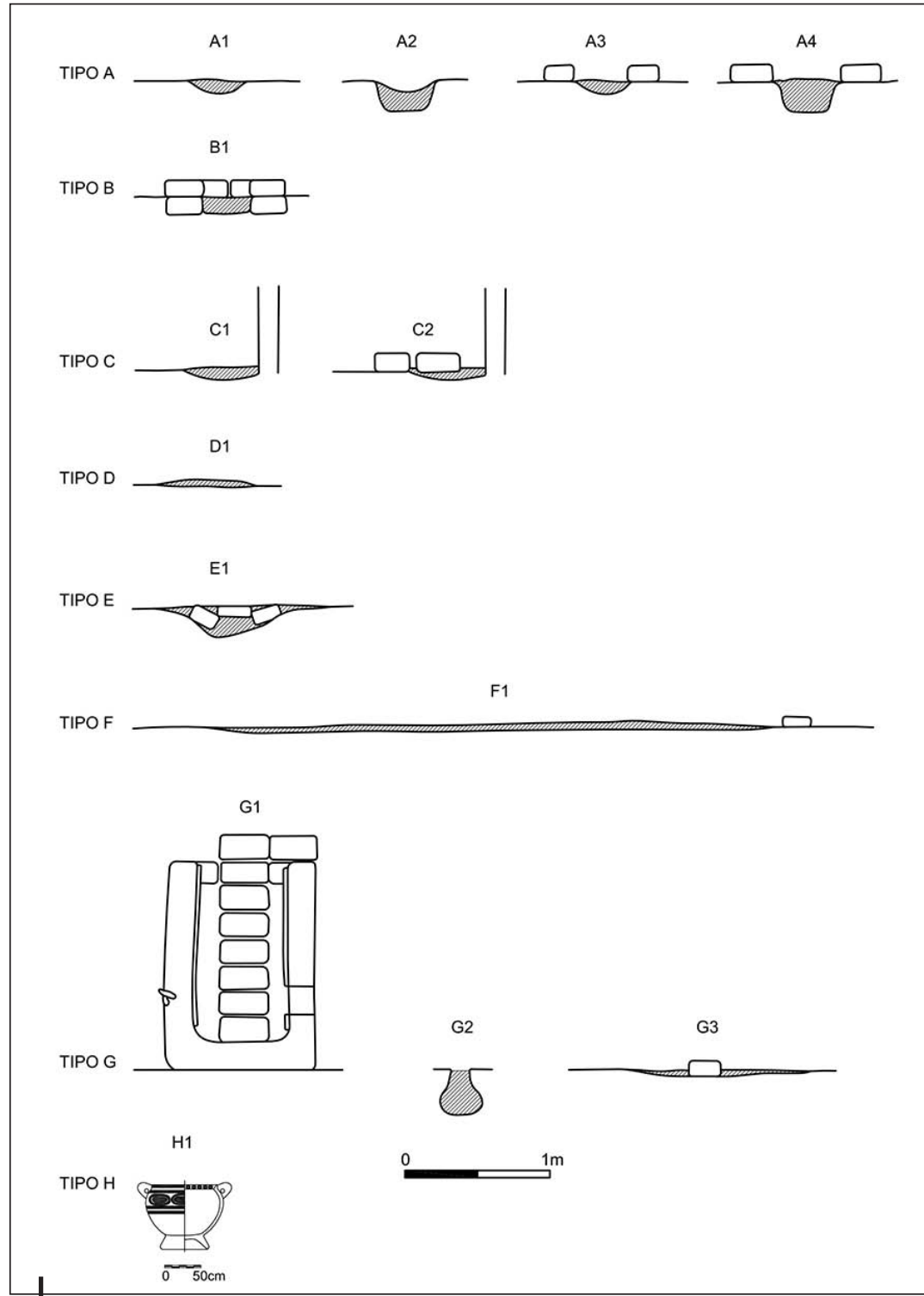

Figura 6 - Esquema con secciones de los tipos de estructuras de combustión Tipo A. Fogón tipo fosa: fogón tipo fosa simple (A1), fogón tipo fosa de paredes verticales (A2), fogón tipo fosa simple con borde (A3) y fogón tipo fosa de paredes verticales con borde (A4); Tipo B. Fogón de estructura rectangular (B1); Tipo C. Fogón adosado al muro: fogón adosado al muro sin borde (C1) y fogón adosado al muro con borde (C2); Tipo D. Fogón plano (D1); Tipo E. Fogón atípico o diverso (E1); Tipo F. Horno alfarero (F1); Tipo G. Horno orfebre: horno de fusión con chimenea (G1), horno de fusión cerrado (G2) y horno de recocido (G3); Tipo H. Brasero (H1)

(c) F. Castillo 


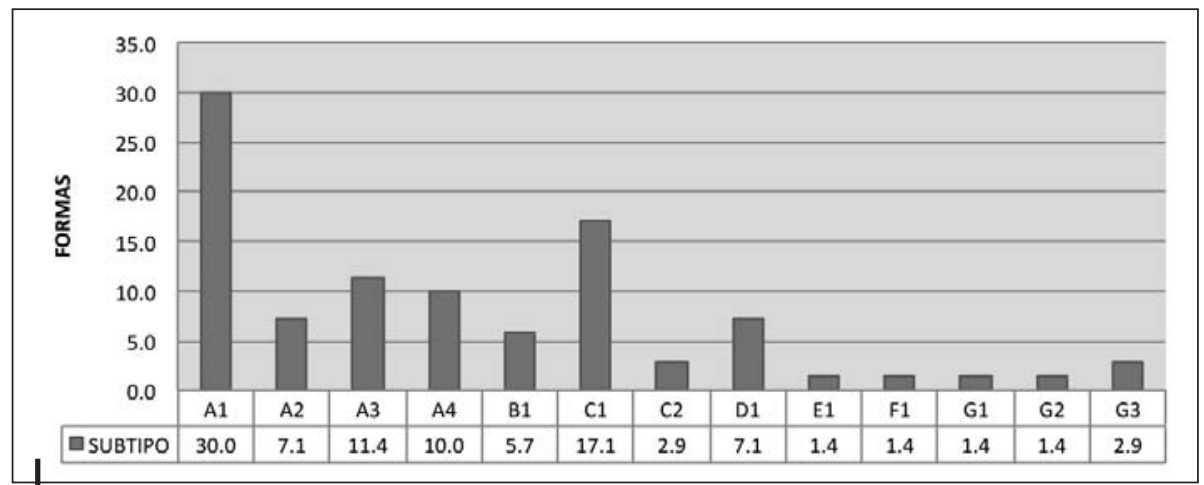

Figura 7 - Porcentaje de las formas según subtipo registradas en el total de la muestra

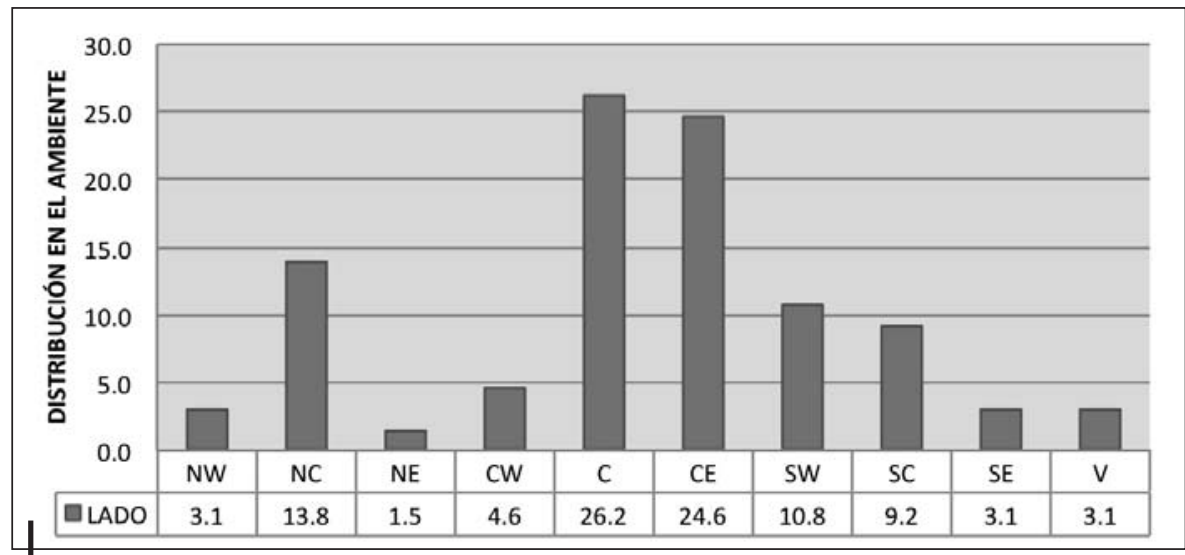

Figura 8 - Distribución porcentual de los fogones al interior de los ambientes o espacios contenedores

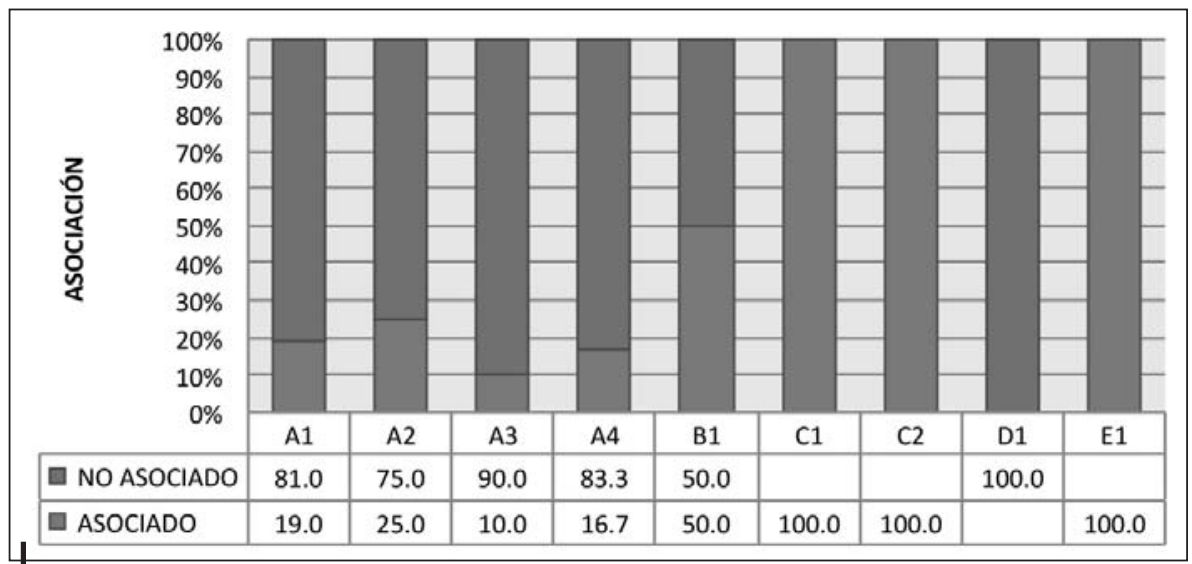

Figura 9 - Porcentaje de los fogones según su asociación a los muros del espacio contenedor 


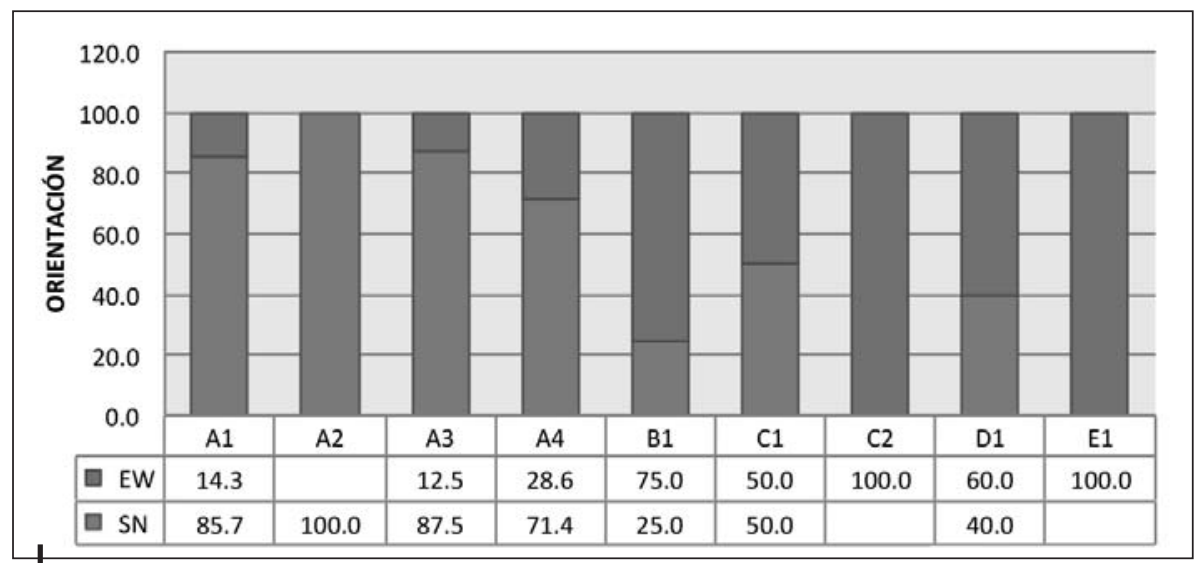

Figura 10 - Porcentaje de los fogones según la orientación del eje mayor

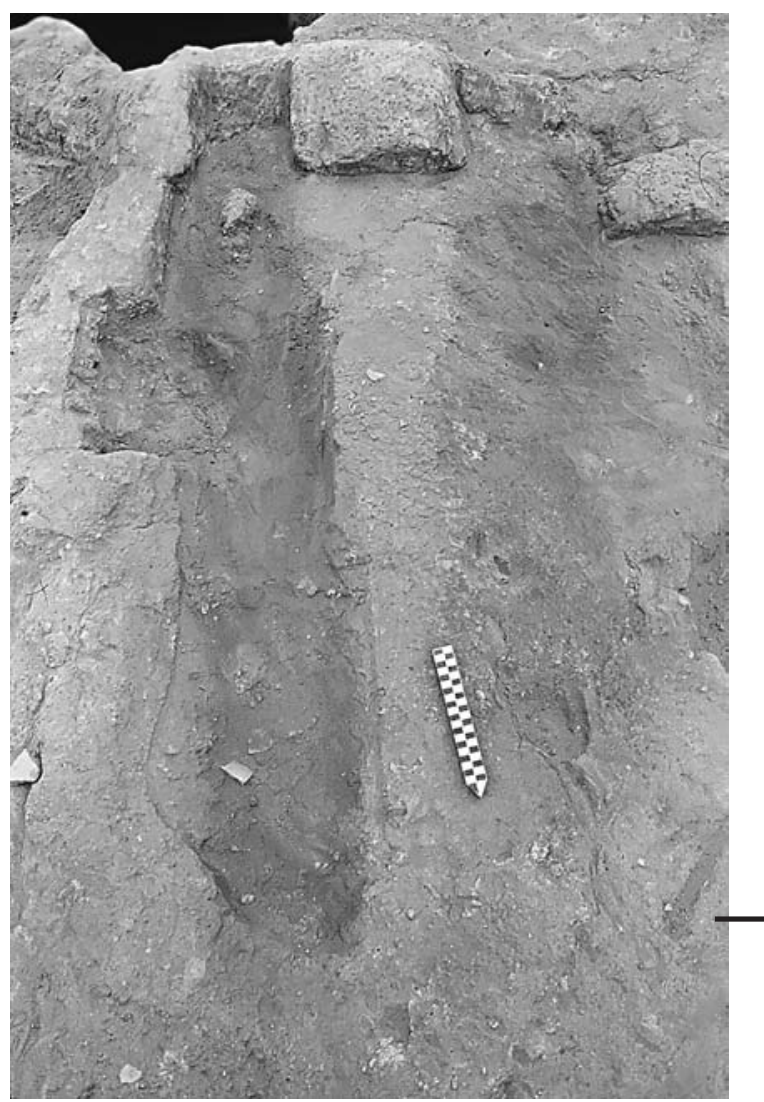

Figura 11 - Vista norte-sur de los fogones de subtipo A1 (fg18 y fg19) registrados en el ambiente 35-11/12 
Subtipo A2: fogón tipo fosa de paredes verticales

Este subtipo se diferencia del anterior por presentar una fosa de mayor profundidad (hasta 0,40 m). Particularmente son de planta rectangular con bordes cortados (figs. 6 y 12); sin embargo notamos casos tardíos, asociados a niveles superiores con planta elíptica u ovoide. Vista de perfil la fosa presenta las paredes ligeramente verticales con un fondo cóncavo. La totalidad de la muestra analizada está orientada de sur a norte; sin embargo, los fogones tardíos están particularmente orientados de este a oeste. Además en tres casos se ha definido áreas de evacuación de ceniza al sur. La muestra estudiada está representada por 5 casos, equivalentes al 7,1\% (fig. 7; ver cuadro 1).

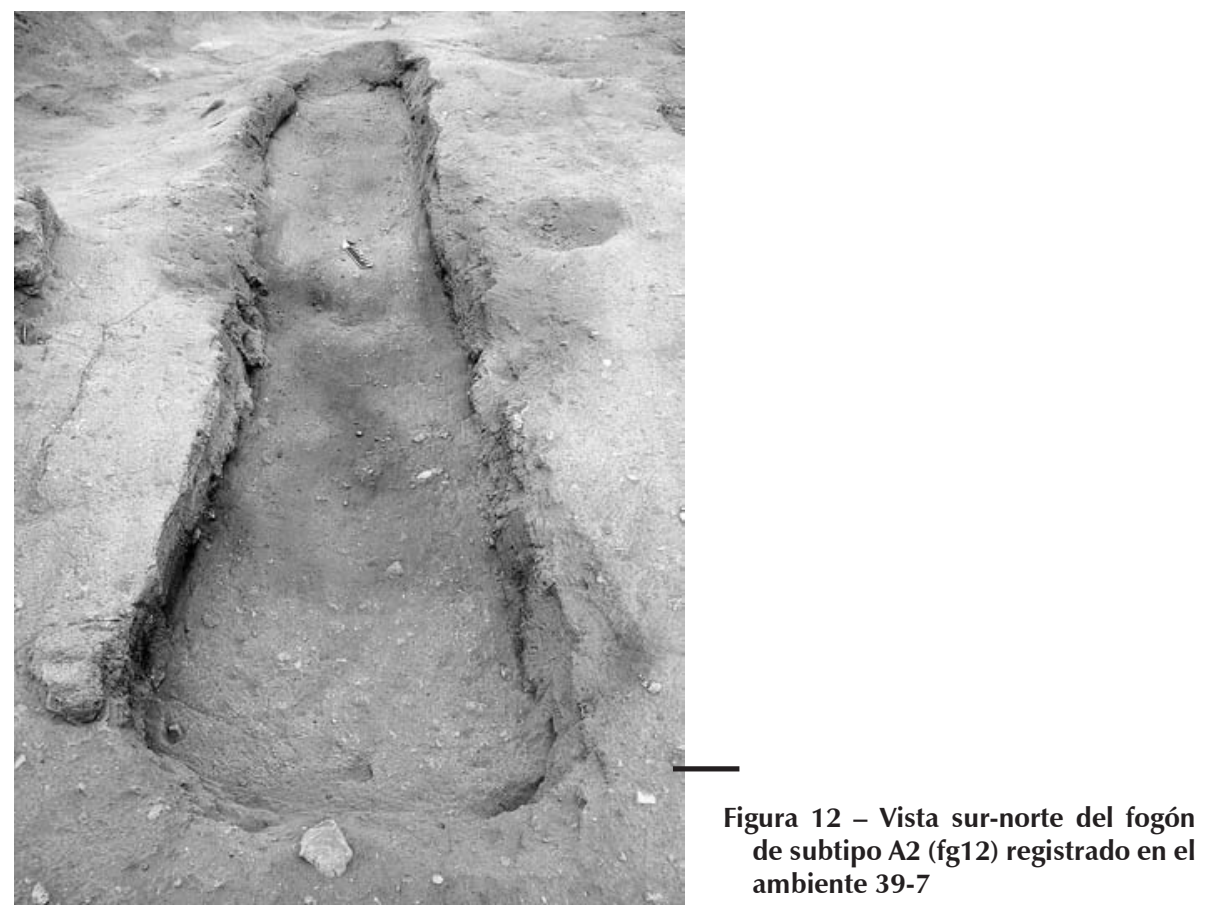

Subtipo A3: fogón tipo fosa simple con borde

Se trata de un fogón excavado en el piso que forma una fosa o cubeta de poca profundidad (posiblemente una variante del subtipo A1). La fosa tiene una planta variable, entre elíptica y rectangular cuyo eje mayor está orientado típicamente de sur a norte (figs. 6 y 13). Se ubica generalmente en el centro del ambiente contenedor y en la mayoría de casos presentan un área de evacuación de ceniza al sur.

Se han recuperado un total de ocho casos, equivalentes a 11,4\% (fig. 7; ver cuadro 1). En todos los casos la fosa presenta dos filas de adobes asentadas sobre el piso del ambiente a lo largo del eje mayor de la fosa. Los adobes pueden estar dispuestos de cabeza y/o soga, de acuerdo al número de filas de adobes utilizado y están 


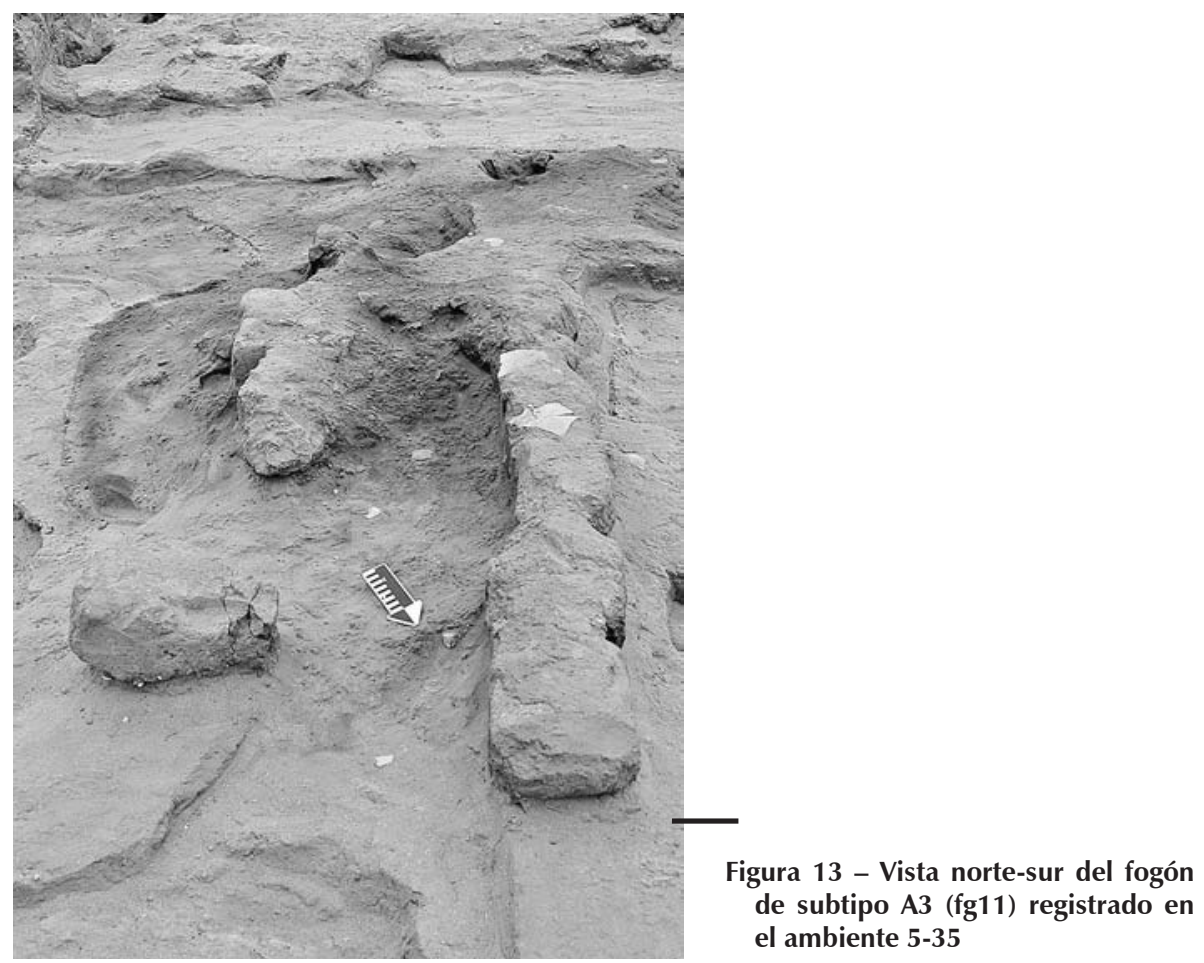

unidos con argamasa de barro y pachilla de fragmentos de cerámica. Los adobes pueden variar en tamaño y es muy típico notar el uso de adobes refractarios.

\section{Subtipo A4: fogón tipo fosa de paredes verticales con borde}

Este subtipo arquitectónicamente es muy similar al subtipo A3, diferenciándose solo por la profundidad de la fosa, esto lo convierte en una variante del subtipo A2 (figs. 6 y 14). Este subtipo presenta el área de evacuación tanto al sur como al norte. Este subtipo está representado por 7 casos, equivalentes al $10 \%$ del total de la muestra (fig. 7; ver cuadro 1).

\section{Tipo B: fogón de estructura rectangular}

Se caracteriza por presentar una estructura rectangular de adobes dispuestos de cabeza asentados en una pequeña plataforma sobre el piso (figs. 6 y 15). Este fogón se ubica al centro del espacio contenedor, cuyo eje mayor está orientado generalmente de este a oeste. El interior de la estructura funciona como cámara de combustión (vista las alteraciones térmicas observadas sobre las paredes); mientras los bordes y fondo están parcialmente oxidados. En ninguno de los casos se han registrado áreas de evacuación de ceniza anexa. La muestra está compuesta por 4 casos, equivalentes al 5,7\% (fig. 7; ver cuadro 1). 
Feren Castillo Luján, Santiago Uceda Castillo, Ramiro Javier March
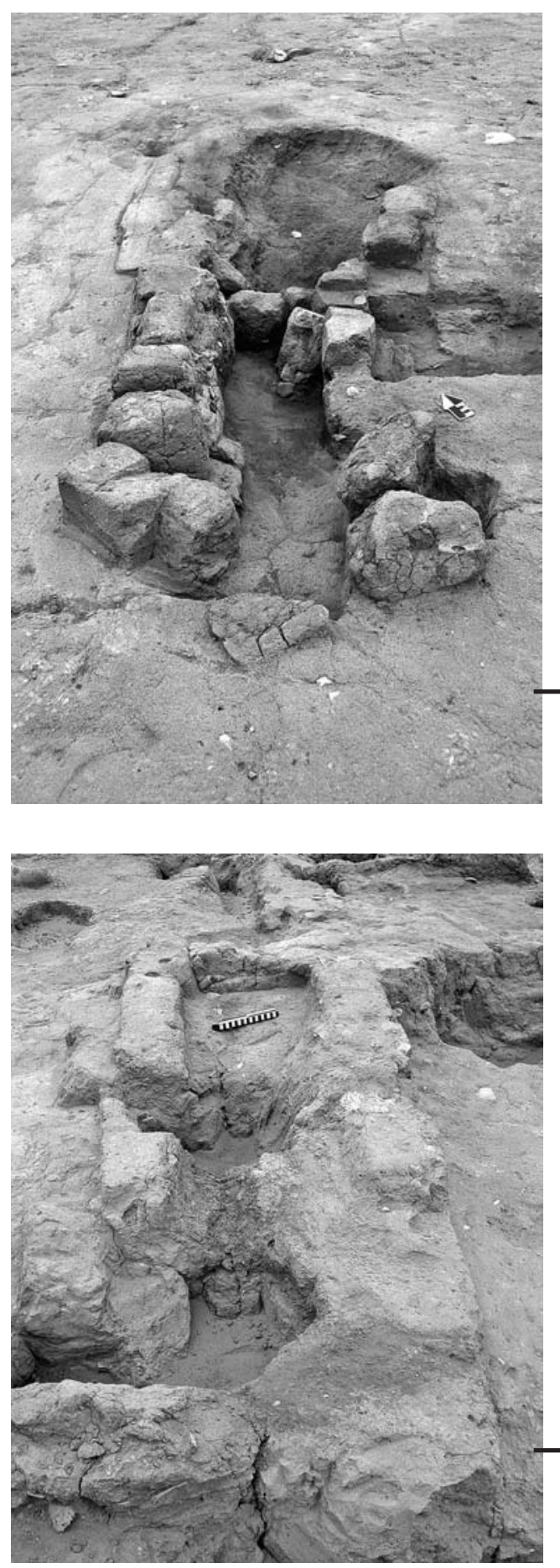

Figura 14 - Vista sur-norte del fogón de subtipo A4 (fg8) registrado en el ambiente 39-8

Figura 15 - Vista oeste-este del fogón de subtipo B1 (fg6) registrado en el ambiente 27-9 


\section{Tipo C: fogón adosado al muro}

Este tipo fue diferenciado a partir de su asociación o adosamiento a los muros de los espacios contenedores. La asociación al muro es totalmente funcional, este funciona como chimenea y ayuda a evacuar los gases producidos en la combustión. Se han identificado catorce casos distribuidos en dos subtipos: (C1) fogón adosado al muro sin borde y (C2) fogón adosado al muro con borde.

\section{Subtipo C1: fogón adosado al muro sin borde}

Este subtipo se caracteriza por presentar una fosa poco profunda, particularmente de planta semicircular adosada a uno de los muros del ambiente que la contiene (figs. 6 y 16). La orientación de este subtipo es igual tanto de este a oeste como de sur a norte. Su asociación a los muros no es recurrente, puede estar en cualquiera de los cuatro muros que definan el espacio contenedor. El muro por lo general se encuentra oxidado, posiblemente por las altas temperaturas alcanzadas durante la combustión que hayan transformado el material o posiblemente se hayan empleado adobes refractarios. La muestra está conformada por doce casos, que equivalen al 17,1\% del total de la muestra (fig. 7; ver cuadro 1).

\section{Subtipo C2: fogón adosado al muro con borde}

Este subtipo podría ser una variante del subtipo anterior. Se diferencia por presentar borde de adobes adosados al muro (figs. 6 y 17). Los 2 casos registrados en la muestra - 2,9\% - (fig. 7; ver cuadro 1), se encuentran adosados al muro norte del ambiente contenedor y su eje mayor está orientado de este a oeste. Al igual que el caso anterior, el muro presenta huellas de la acción del fuego (estado de oxidación y hollín).

\section{Tipo D: fogón plano}

Se trata de un tipo de fuego al aire libre, conocido actualmente como: fogata. Este tipo se caracteriza por estar sobre el piso del ambiente contenedor, que a menudo puede ser confundido como lentes de ceniza asociados a los rellenos de una ocupación. Sin embargo, se pueden distinguir al apreciar formas elípticas de suelo termo alterado, con orientación diversa y ubicados al centro del espacio contenedor (figs. 6 y 18).

Este tipo de fogón no está documentado en la mayor parte de la muestra, posiblemente fueron descritos como simples «quemas» asociadas al piso; sin embargo, hemos logrado identificar cinco casos, equivalentes al 7,1\% (fig. 7; ver cuadro 1).

\section{Tipo E: fogón atípico o diverso}

En esta categoría hemos optado por incluir a los fogones cuyas formas no se incluyen en los tipos anteriores. En la muestra se ha registrado un solo caso atípico (fig. 7; ver cuadro 1). Sin embargo, existen otros casos únicos registrados en otros conjuntos y sectores del sitio, que no han sido considerados en nuestra muestra. 
Feren Castillo Luján, Santiago Uceda Castillo, Ramiro Javier March
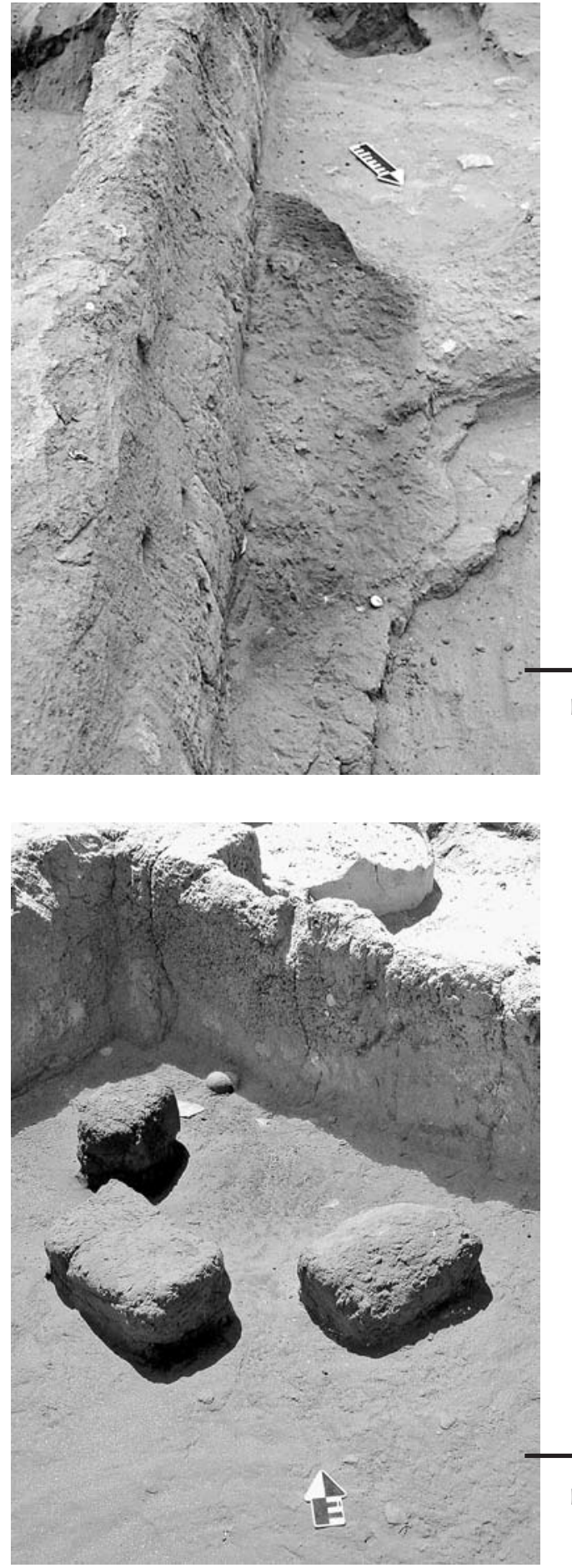

Figura 16 - Vista norte-sur del fogón de subtipo C1 (fg17) registrado en el ambiente 5-22

Figura 17 - Vista sur-norte del fogón de subtipo C2 (fg15) registrado en el ambiente 5-7 


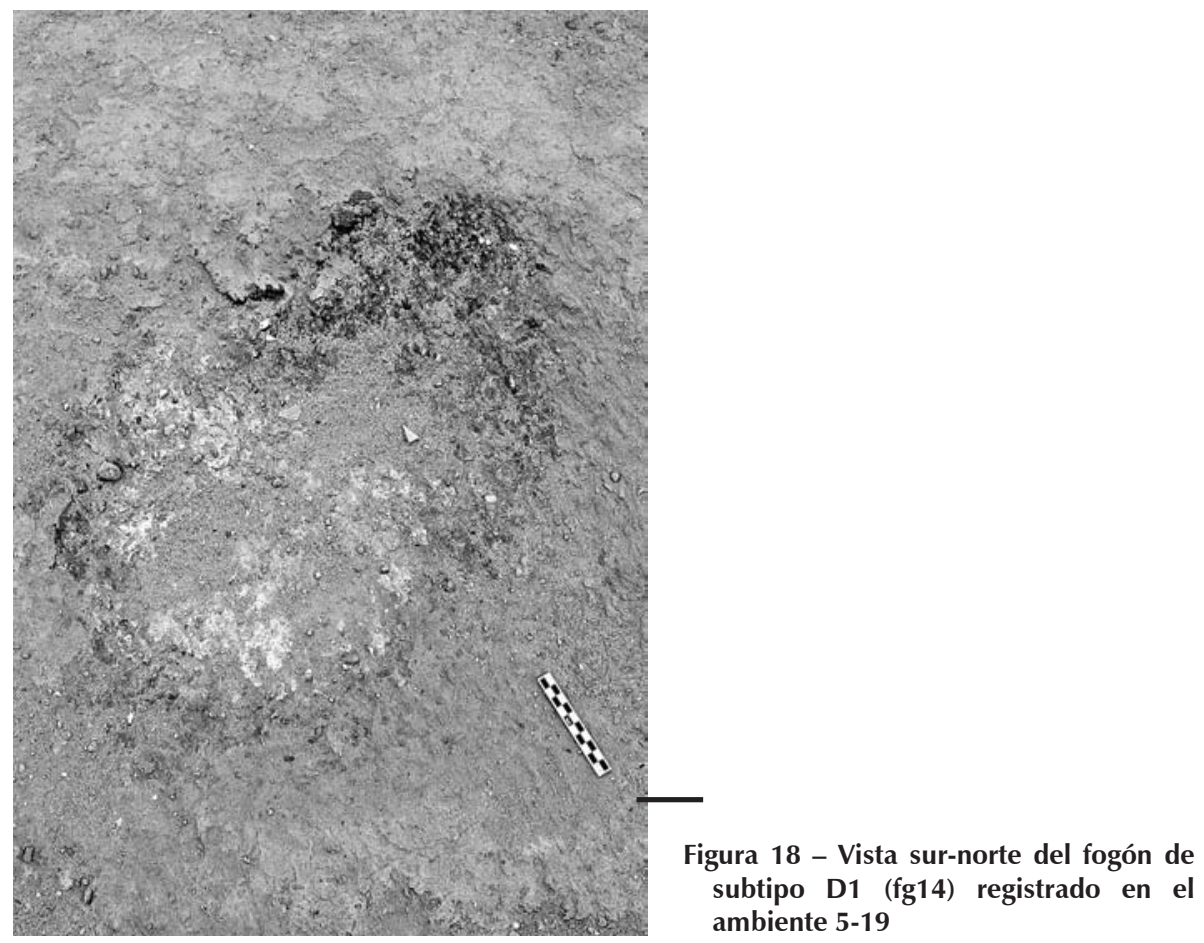

Se trata de una fosa de planta elíptica y corte cóncavo, cuyo eje mayor está orientado de este a oeste (figs. 6 y 19). Se diferencia por presentar adobes refractarios al interior de la cámara de combustión y por debajo del nivel del piso. Además tiene un adobe en la parte superior que forma una bóveda, posiblemente presentaba pequeñas aberturas en la parte superior que eran utilizadas para que salga la lumbre y dé directamente en las bases de las ollas. En el lado este se ubica la entrada de combustible, mientras en la parte contraria el área de evacuación de residuos.

\section{1. 2. Los hornos}

Los hornos son un tipo de dispositivo empleado para alcanzar altas temperaturas que permiten transformar materiales como la arcilla, y para transformar elementos químicos sólidos como los metales. Los hornos son muy variados y están dispersos en todo el mundo. En nuestro caso, se han identificado dos tipos: horno alfarero (tipo F) y horno orfebre (tipo G).

\section{Tipo F: horno alfarero}

Este horno se ubica en un espacio grande y abierto (posiblemente sin techar). La forma de la planta es generalmente circular con ligeras irregularidades en sus bordes por los procesos de posabandono (figs. 6 y 20). El horno presenta una 
Feren Castillo Luján, Santiago Uceda Castillo, Ramiro Javier March

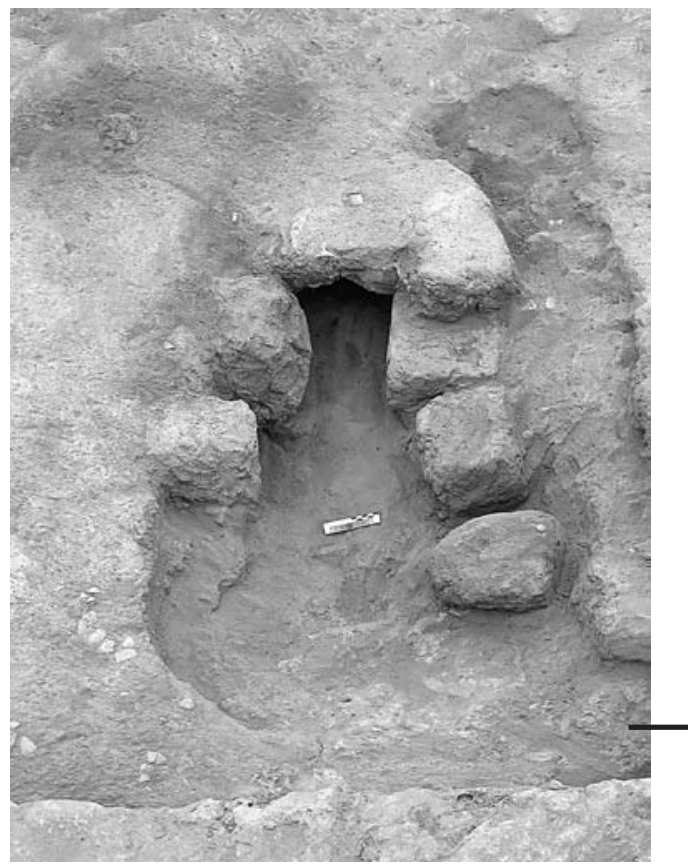

Figura 19 - Vista este-oeste del fogón de subtipo E1 (fg6) registrado en el ambiente 27-9

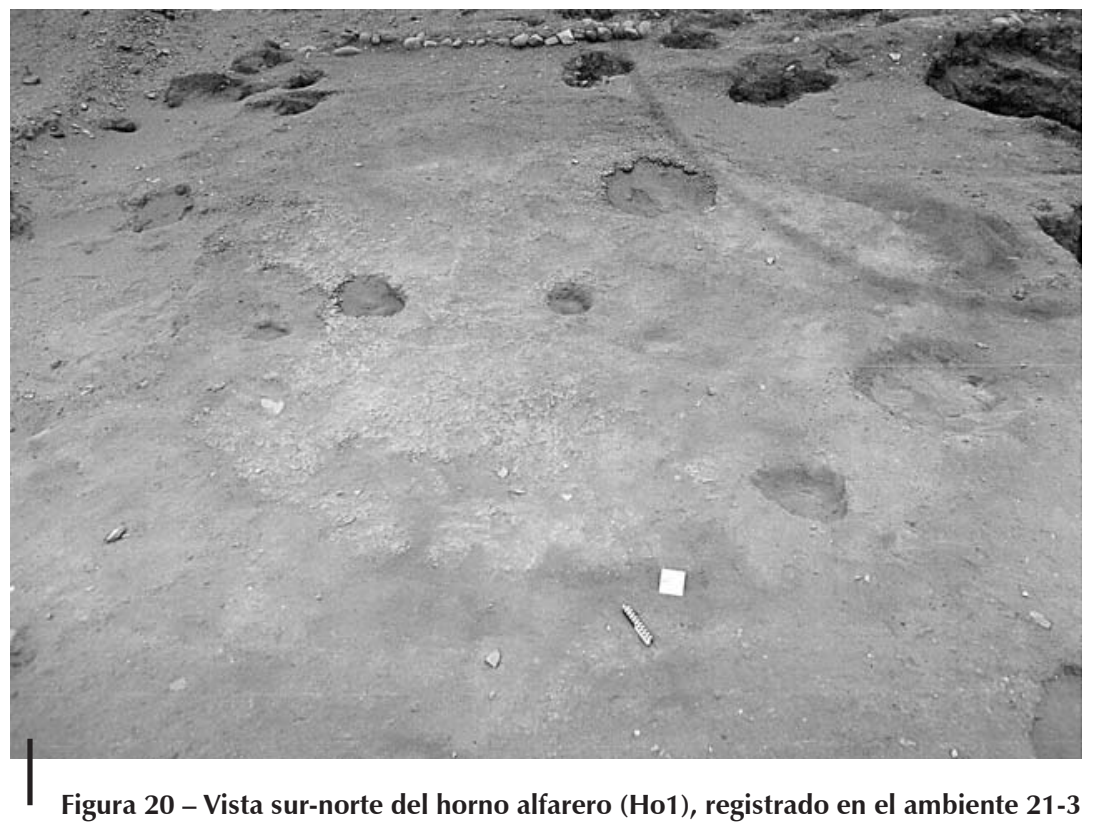


cavidad no muy profunda y contiene un relleno de ceniza mezclado con material cultural de color grisáceo en sus límites y marrón rojizo en la parte central.

La muestra estudiada identificó un solo tipo de horno de alfarero Moche (Ho1) en el CA21, equivalente al 1,4\% del total de la muestra (fig. 7; ver cuadro 1); sin embargo, la evidencia en el sitio - aún reducida - nos presenta un total de cinco casos distribuidos en los dos talleres alfareros (CA21 y TAM).

\section{Tipo G: horno orfebre}

Los hornos orfebres son estructuras de combustión muy diversa diseñadas para soportar altas temperaturas $\left(1000-1200{ }^{\circ} \mathrm{C}\right)$. En su interior tiene lugar la fusión o recocido del mineral en metal. La muestra incluye todos los casos - hasta la fecha - reportados para el sitio. Estos se encuentran distribuidos en el CA7 (Ho1) y CA27 (Ho1, Ho2 y Ho3). El presente estudio nos ha llevado a clasificarlos en horno de fusión con chimenea (G1), horno de fusión cerrado (G2) y horno de recocido (G3).

\section{Subtipo G1: horno de fusión con chimenea}

Este horno está compuesto por una chimenea de forma circular cuya construcción se hizo posible superponiendo nueve filas de adobes de manera circular, obteniendo un diámetro de 0,55 m y una altura de 1,20 m (figs. 6 y 21). Cerca de la base, el horno presentaba una pequeña abertura, que estaba tapada por un adobe esculpido. En el mismo nivel de la abertura presenta fragmentos de cerámica entre los adobes que sirvieron para controlar la temperatura (Fraresso, 2007).

Internamente presentaba una capa de arena eólica de 0,60 m de espesor sobre una capa de arena mezclada con carbones de madera y pequeños fragmentos de mortero enrojecido por el calor. Los últimos 0,20 m eran más compactos, contuvieron más carbones y fragmentos oxidados de mortero mezclados con ceniza. El paramento interno de la estructura circular se encontraba altamente termo alterado, siendo más prominente en la parte inferior. Se trata de un caso único, equivalente al 1,4\% (fig. 7; ver cuadro 1), registrado en el CA7 (Chapdelaine, 1998).

\section{Subtipo G2: horno de fusión cerrado}

Este horno debió ser una pequeña fosa revestida por una gruesa capa de arcilla fina sin aditivos con una bóveda elaborada mediante aplicaciones sucesivas de paneles de arcilla y canales a manera de chimeneas en la parte superior (fig. 6). La presencia de fragmentos de cerámica de pasta gruesa con restos de metal en vitrificaciones respalda la hipótesis.

Este subtipo no se ha conservado adecuadamente en el taller orfebre - CA27(ver cuadro 1). Sin embargo debió tratarse de aquella estructura adosada al muro del ambiente 27-30. Este horno orfebre (Ho1) habría tenido la forma de aquellos registrados en Yécala (Makowski \& Velarde, 1998) o Batán Grande (Shimada, 1994a). 


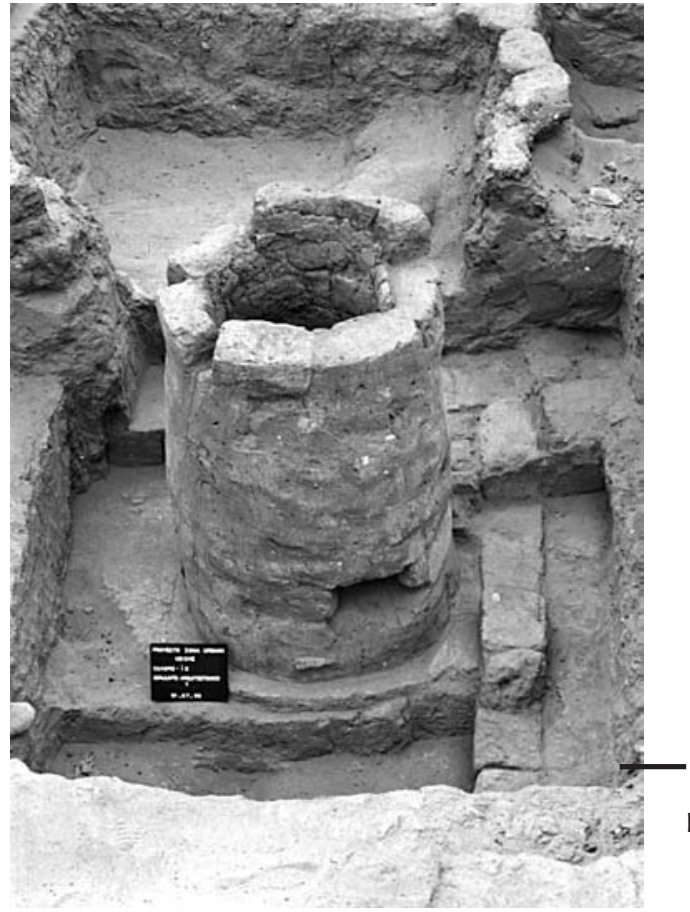

Figura 21 - Vista sur-norte del horno de subtipo G1 (Ho1), registrado en el ambiente 7-15

Subtipo G3: horno de recocido

Estos hornos están elaborados en el suelo, a manera de fogones del tipo fosa simple, de planta circular y pequeñas dimensiones (figs. 6 y 22). En la parte central de la estructura presentaban un adobe refractario. Estos hornos podrían confundirse con fogones, pero la presencia de restos de escoria, prills y toberas podría ayudar con su identificación. En la muestra podemos distinguir dos hornos de recocido en el taller orfebre $\mathrm{Ho} 2$ y Ho3, equivalentes al 2,4\% del total de la muestra (fig. 7; ver cuadro 1).

\section{1. 3. Los braseros}

Los braseros fueron recipientes inmuebles hechos de cerámica donde se contenían brasas para ser trasladadas a cualquier dirección. Estos fueron vasijas de cerámica como los cuencos semiesféricos de base pedestal con o sin agarraderas tipo anillo a la altura del borde.

\section{2. Las concentraciones de ceniza}

Las concentraciones de ceniza son acumulaciones de cenizas y/o carbones que en ocasiones se encuentran mezclados con restos orgánicos (óseos, malacológicos y vegetales) y no orgánicos (lítico, fragmentos de adobes quemados, metales y 


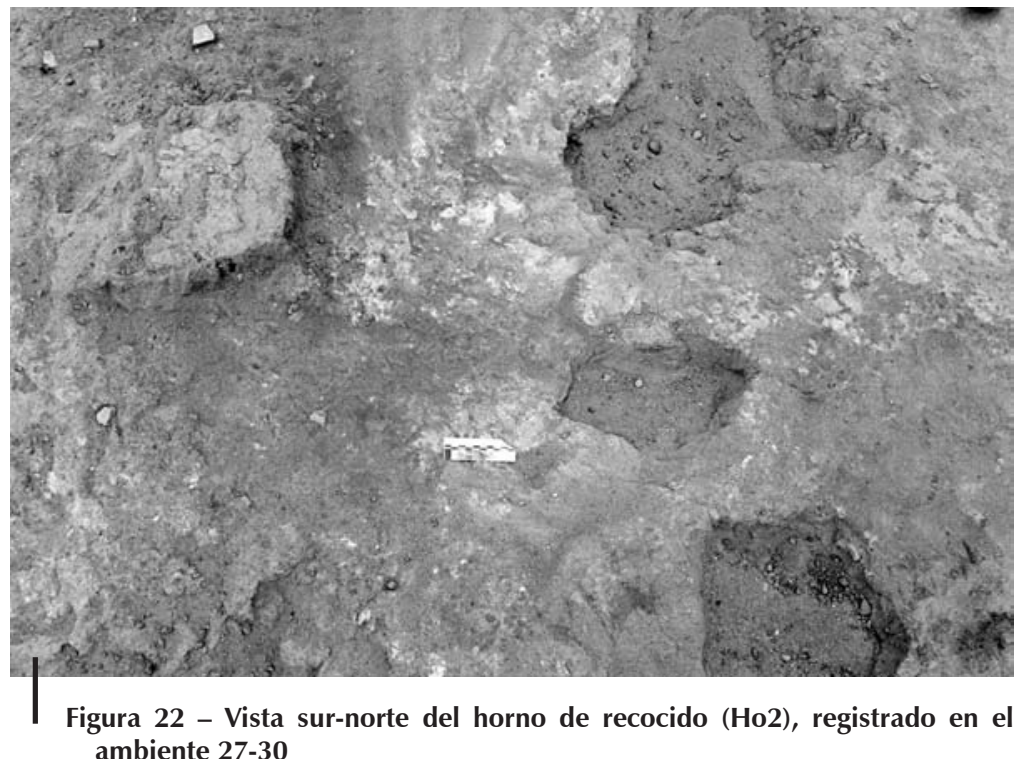

cerámica). Estos podrían tratarse de depósitos de cenizas frías provenientes de la limpieza de los fogones. En la muestra hemos determinado 35 casos (ver cuadro 1). Estas se diferencian de las estructuras de combustión por ser muy irregulares y podrían ser áreas de desechos, botaderos o evacuación de ceniza que se generan en los diferentes tipos estructurados o incluso áreas de actividades relacionadas con el uso de las cenizas (fig. 23). El estudio demuestra que las concentraciones de ceniza no presentan suelos claramente alterados térmicamente u oxidados; sin embargo existen muchos casos de fogones abandonados y convertidos en áreas de desechos, lo cual puede dificultar la identificación de estos en un principio. Un caso particular fue registrado en el CA5, en un principio se creía que era un fogón (cc10); sin embargo el análisis espacial nos indica que fue abandonado y convertido en área de desecho (fig. 2).

La mayoría de los casos corresponden a pequeños ambientes (depósitos) reutilizados como botadores. Sin embargo, un buen porcentaje corresponde a áreas de evacuación asociadas directamente a los fogones tipo fosa. Es importante registrar las áreas de evacuación como elementos independientes y distintos a la matriz de los fogones, pues el estudio arqueométrico podría revelarnos detalles sobre la microtemporalidad del depósito.

\section{3. Los testigos de incendio}

Los testigos de incendio son evidencias de paramentos oxidados o rasgos arquitectónicos quemados, tanto de espacios domésticos como de grandes 


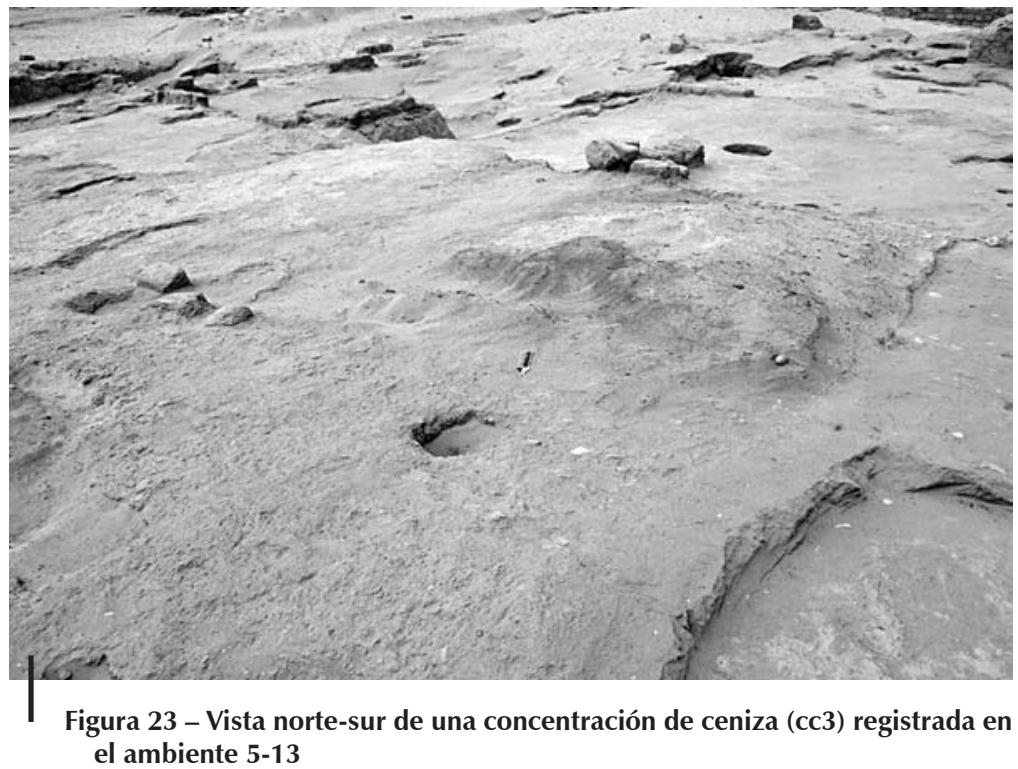

edificios o templos. La muestra estudiada solo permitió identificar este tipo de evidencias en dos paramentos del Templo Viejo de Huaca de la Luna (ver cuadro 1). Los dos casos estudiados, a simple vista son totalmente distintos. El primero se trataría de una acumulación de combustible que permita consumirse totalmente como hojas o textiles. La quema «ritual» de estos desechos debió producir la alteración térmica del paramento (fig. 24). El segundo, en cambio, presenta evidencias de haberse quemado el techo (Gynerium sagitatum «caña brava»). Se puede apreciar in situ restos del techo quemado, así como improntas de cielo rasos totalmente oxidados (fig. 25).

La quema de arquitectura ha sido reportada para otros sitios Moche como Pampa Grande (Shimada, 1994a) y Cao Viejo (Quilter, 2008), relacionándola al abandono o clausura de un edificio (Guffroy et al., 1994). Sin embargo, recientes investigaciones nos muestran que este tipo de quemas podrían ser interpretadas como un praxis ritual que connota actos de limpieza o purificación de los espacios arquitectónicos (Chávarri \& Mejía, 2011).

\section{DISCUSIÓN Y COMENTARIOS GENERALES}

\section{1. Los fogones permanentes y temporales}

Los fogones fueron empleados para la cocción de alimentos y elaboración de bebidas (chicha); además es posible que estos estén asociados a actividades complementarias como la de integrar las relaciones sociales de la familia en rituales de culto. 


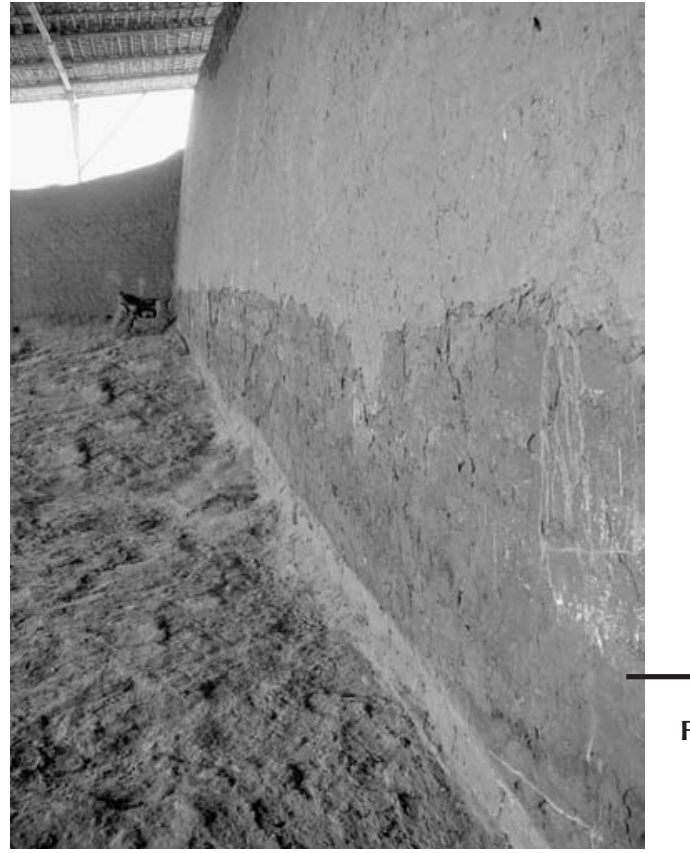

Figura 24 - Vista sur-norte del testigo de incendio 2 registrado en la terraza 2 del último edificio del Templo Viejo de Huaca de la Luna

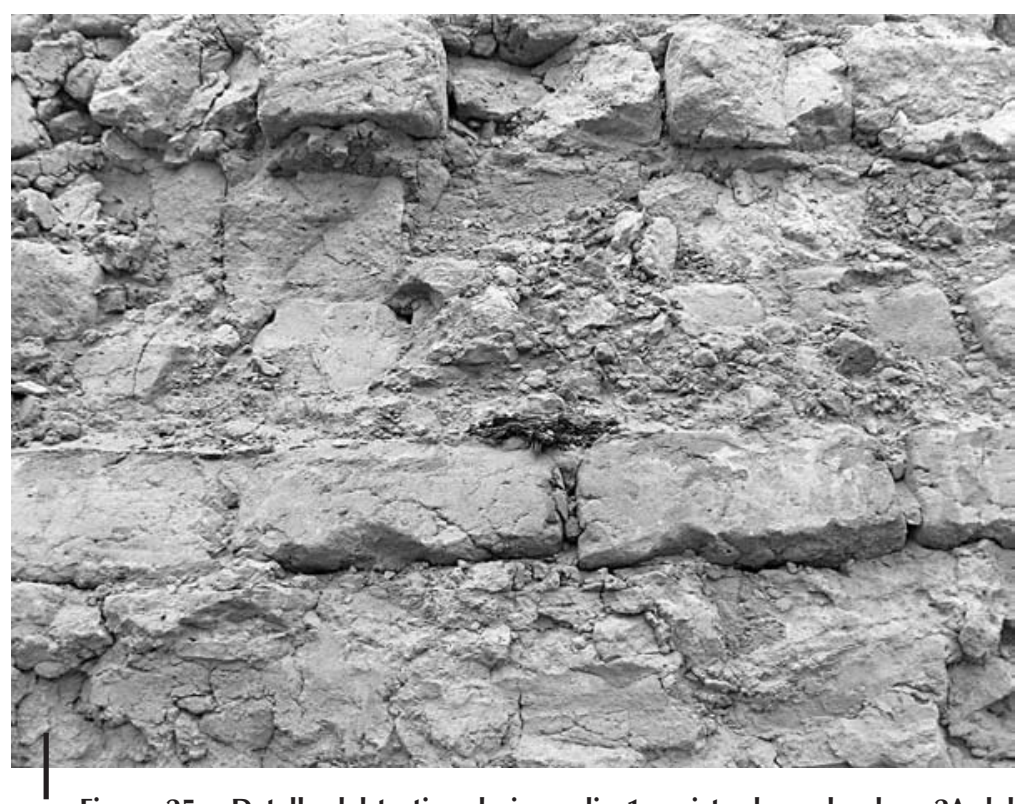

Figura 25 - Detalle del testigo de incendio 1 registrado en la plaza $2 \mathrm{~A}$ del Templo Viejo de Huaca de la Luna 
Los fogones del tipo A son los más abundantes en nuestro estudio, por lo que optamos por hacer hincapié en el tamaño, según el eje mayor. El 31,7\% de la muestra son medianos, entre 1,01 y 1,50 m, seguido por los pequeños menores a $1 \mathrm{~m} \mathrm{(26,8 \% ),} \mathrm{grandes} \mathrm{entre} \mathrm{1,51} \mathrm{y} 2 \mathrm{~m}(17,1 \%)$, muy grandes entre 2,01 y 2,50 m $(14,6 \%)$ y en menor proporción extra grandes mayores a 2,51 m (9,8\%). Estas proporciones nos hacen sugerir que existe una estandarización de este tipo entre el 1,01 m y 1,50 m. Con respecto al eje menor, se tomó en cuenta el ancho de la cámara de combustión. Este varía entre los 0,30 y 0,70 m y es irregular con respecto a la dimensiones del eje mayor. Existe la posibilidad de que mientras más ancha era la cámara, mayor era el tamaño de las vasijas que se empleaban en la cocción y, por lo tanto, empleaba más cantidad de combustible. Esto denota una alta producción de alimentos y/o bebidas.

Los diferentes casos conservados de fogones del tipo A permiten identificar cuatro partes arquitectónicas: (1) La fosa, que funciona como cámara de combustión donde se coloca el combustible sólido (leña) y es quemada en el proceso de ignición; (2) Las estructuras de adobes, que sirven para sostener vasijas de gran tamaño y preparar alimentos y/o bebidas; (3) La entrada de combustible, es la parte por donde la persona puede avivar el fuego introduciendo más combustible o aire; (4) El área de evacuación, que se ubica al otro extremo de la entrada de combustible, por lo general sirve para evacuar la ceniza, restos de carbón o comida que se acumulan al interior de la cámara de combustión.

Los fogones de Tipo B son poco comunes, pero imponentes al interior de las cocinas del núcleo urbano. Particularmente están asociados a cocinas con presencia de vasijas in situ de gran tamaño (tinajas), lo que sugiere su función exclusiva para la preparación de chicha; además sus dimensiones (mayores a 2 m de largo) respaldan su asociación a la producción masiva de bebidas.

Con respecto a los fogones de Tipo $\mathrm{C}$, doce casos fueron registrados en posibles áreas de cocción de alimentos y/o bebidas, mientras dos casos estuvieron asociados a un patio con banqueta, lo cual sugiere que fueron empleados como espacios temporales para calentar alimentos $y / u$ otra actividades que deben ser aclaradas en futuras investigaciones.

Otro caso particular son los fogones de Tipo D, todos los casos registrados no estaban asociados a alguna cocina, sino a patios o antesalas. Esto sugiere la posibilidad de haber sido empleado como calefacción, alumbramiento u otra actividad desconocida. Sin embargo, no se puede descartar el uso secundario de cocción de alimentos hasta no realizar un estudio químico de los sedimentos que demuestren la ausencia de esteroles de animales (March et al., 1989). De esta manera nuestra propuesta ha sido elaborada desde una perspectiva netamente arquitectónica, cuya función específica deberá ser corroborada en futuras investigaciones analíticas².

2 Las últimas investigaciones al interior del templo viejo de huaca de la Luna están reportando una diversidad de fogones planos y atípicos que merecen también su atención en futuras interpretaciones. 
A partir del trabajo etnográfico elaborado en cocinas modernas en los valles de Moche y Virú (Castillo, 2012), podemos sugerir que los fogones del subtipo A1, A2 y C1 serían fogones temporales y empleados en eventuales celebraciones para abastecer un mayor número de comensales o asistentes al interior de las residencias Moche. Estos debieron ser, en su estado original (antes del abandono), como los fogones del subtipo A3, A4 y C2 respectivamente. El dato etnográfico nos hace pensar que las cocineras preparaban el espacio donde colocarían sus fogones temporales, teniendo en cuenta la ventilación, iluminación y facilidad de circulación al interior de la cocina (fig. 26).

En el caso que la cocina contara con un fogón permanente, este sería el de mayor importancia y el que mayor grado de alteración térmica presente en los adobes a causa de la constante combustión (fig. 27). Sin embargo, es de notar en los fogones modernos que los adobes solo logran oxidarse en la cara interna de la cámara de combustión; mientras los fogones moches, en su mayoría, presentan los adobes totalmente oxidados.

¿Se puede sugerir un posible conocimiento, por parte de los Moche, del manejo y control del calor al emplear adobes refractarios? De ser así, habría que plantear la posibilidad de un espacio en la ciudad Moche destinado a la elaboración de adobes crudos y en menor escala quemados o refractarios. La evidencia en los hornos alfareros registrados hasta la fecha demuestra que fueron básicamente para cerámica ritual (Uceda \& Armas, 1997) y abalorios (Chiguala et al., 2007), mas no para cocción de adobes. Otra alternativa podría ser la preparación de estos adobes al interior de los mismos fogones; esto deberá ser verificado en futuras investigaciones experimentales. Para finalizar el tema de los adobes refractarios, es evidente también la reutilización de estos en cámaras funerarias (Zavaleta et al., 2011) y como parte del montaje o remodelación de muros de las viviendas Moches (Meneses et al., 2012).

\section{2. Los hornos alfareros de Moche}

El trabajo etnográfico realizado en Mórrope (Shimada, 1994b) sugiere que los hornos suelen encontrarse en áreas cercanas, algunas veces adyacentes a sus talleres. Los hornos abiertos de Mórrope alcanzan temperaturas por encima de 800 a $850{ }^{\circ} \mathrm{C}$ con la que consiguen la cocción completa de grandes tinajas y pequeñas vasijas. La mayoría de talleres están diseñados para la manufactura de cerámica, pero existe la presencia y uso ocasional de fogones demarcados por adobes que sirven para calentar agua o para realizar actividades culinarias de pequeña escala.

Las vasijas a oxidar deben ser transportadas desde un lugar cercano al horno para ser precalentadas colocándolas sobre un promontorio de estiércol seco o a lo largo de este. La carga del horno se logra colocando leña en el fondo del horno, luego van las vasijas precalentadas en posición vertical y cubiertas completamente con leños de zapote y algarrobo, en la base se colocan tiestos grandes y pesados, 
Feren Castillo Luján, Santiago Uceda Castillo, Ramiro Javier March

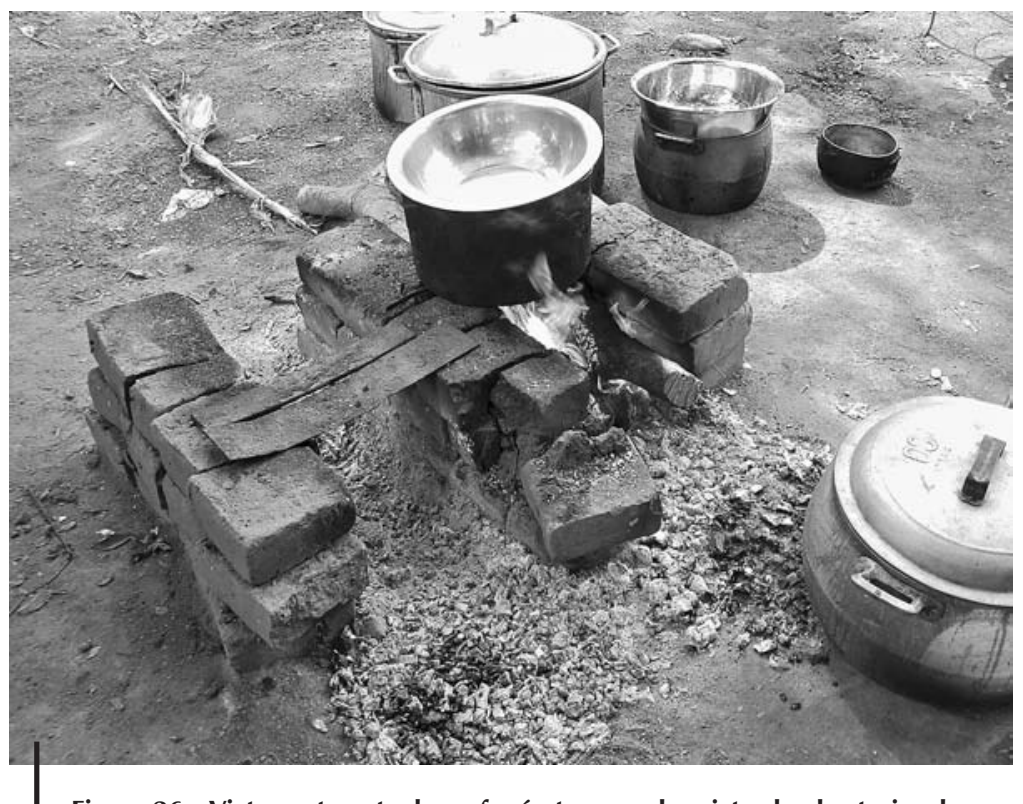

Figura 26 - Vista oeste-este de un fogón temporal registrado al exterior de una vivienda en el sector Huaca Larga (valle de Virú)

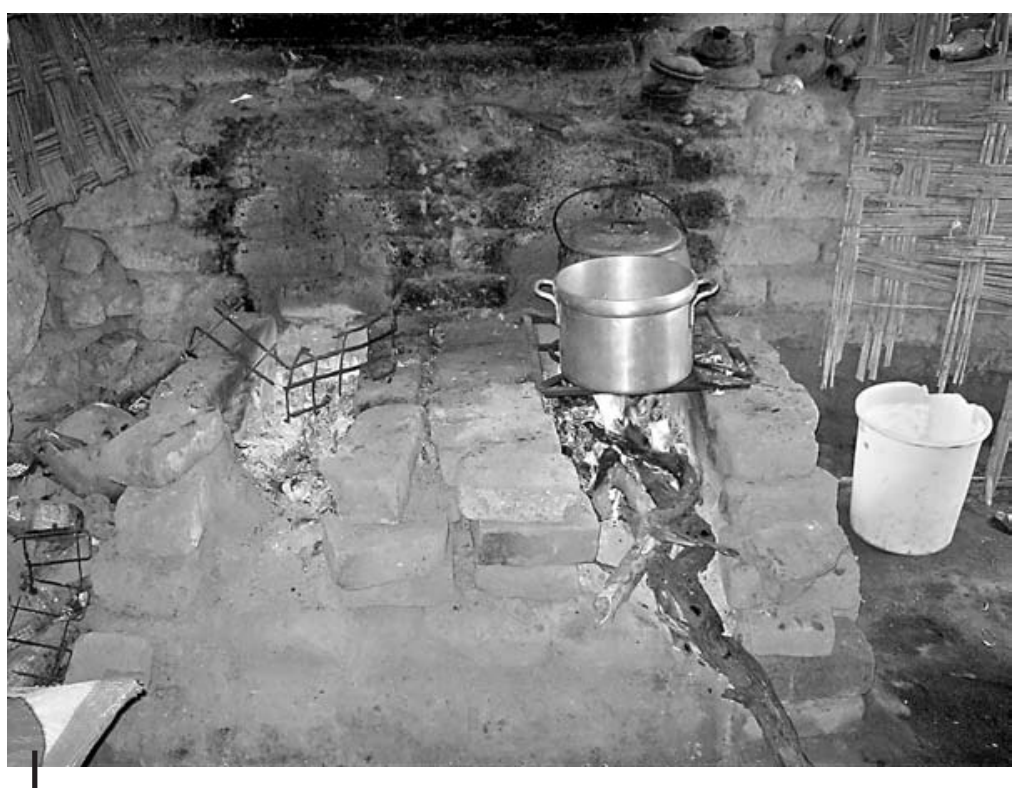

Figura 27 - Vista oeste-este de un fogón permanente al interior de una vivienda en Virú Pueblo (valle de Virú) 
y en la cima solo tiestos pequeños y livianos. Finalmente, la capa de tiestos es cubierta por estiércol, dejando una abertura en los extremos del horno para fines de iluminación.

Los hornos alfareros Moche presentan características similares a las reportadas en Mórrope. Esto respalda que estos hornos fueron abiertos y empleados para transformar objetos de arcilla en piezas de cerámica a gran escala (Uceda \& Armas, 1997; Chiguala et al., 2007).

\section{3. El uso de los hornos orfebres}

Con respecto a los hornos de fusión, los cronistas reportan el uso de sopladores para abastecer de aire el horno donde se funden metales (Benzoni, 1968; De la Vega, 1991). Estos sopladores han sido materializados en la iconografía Moche (Donnan, 1998). El cuenco reportado se caracteriza por figuras modeladas dispuestas alrededor de una estructura en forma de cúpula hueca. Presenta tres personajes usando sopladores para introducir aire dentro del horno; mientras que el cuarto personaje está manipulando uno de los objetos que está encima de la cúpula. En la parte alta del horno se encuentran el carbón, dos objetos en forma de «T» que podrían ser preformas de tocados, lingotes o láminas de metal que están en proceso de fundición o recocido para el trabajo del laminado; además de otros dos objetos redondeados no identificados que podrían ser preformas de máscaras.

Los sopladores habrían avivado el fuego del horno a través de los tubos de caña revestidos con toberas de cerámica, esto con el fin de obtener altas temperaturas. El cuarto personaje tiene un objeto en las manos que posiblemente sirvió para retirar las piezas de metales y llevarlas al martillado. La presencia de otros cuencos con cúpula hueca en su interior descartan la idea de que el horno orfebre haya presentado esa forma (Donnan, 1998).

Esta misma escena está representada en los murales asociados tanto en el tema complejo de Huaca Cao, complejo El Brujo (Franco \& Vilela, 2003), como al reciento esquinero de la Plaza 1 de Huaca de la Luna (Tufinio, 2005). Cabe resaltar que la escena representada en la Huaca de la Luna no es lo suficientemente clara, pero guarda cierta similitud a la representada en la Huaca Cao.

El análisis comparativo de ambas escenas nos demuestra que el personaje de la derecha está soplando a través de un tubo (posiblemente de caña) para avivar el calor dentro de una pequeña estructura en forma de cúpula. Mientras el personaje de la izquierda presenta el brazo izquierdo levantado como en posición de dar golpe. Estos personajes dan la clara impresión de ser el primero un metalurgista fundidor y el segundo un orfebre deformador (Fraresso, 2007).

Las representaciones anteriormente descritas parecen ser similares, las tres representaciones enseñan personajes sopladores; además de un personaje orfebre deformador, el cual sugiere que se habría estado representado un horno de recocido, donde se calentaba el metal para ser luego deformado. En la muestra estos hornos están asociados a un horno de fundición cuya asociación al 
muro sugiere que fue ideal para los orfebres, puesto que habría sido el lugar más oscuro del ambiente y habrían mantenido una mejor lectura del metal fundido (Fraresso, 2010).

\section{4. El fuego portátil}

Hasta la fecha se desconoce el uso de recipientes especiales para este uso, es más, no se ha realizado un análisis del interior de las vasijas para deducir rasgos conservados de ceniza o quema antes del abandono. Partiendo de la información bibliográfica, se ha identificado una representación de uso de fuego portátil en la iconografía mural de la sociedad Moche.

Se trata de un mural polícromo ubicado en el templo de Pañamarca, valle de Nepeña (Bonavia, 1961; 1985). A pesar de la destrucción que ha sufrido el sitio se pudo identificar una escena ritual de sacrificio humano que se describe a continuación.

Esta escena representa una variante del tema de la presentación descrita con mucho detalle por Donnan (1975). Para nuestros fines solo vamos a detenernos en la escena del primer acompañante de la sacerdotisa que lleva la copa con la sangre del sacrificado (fig. 28). Es posible que lo que sostiene en la mano izquierda sea un cuenco con base anular, del cual desprende una flama y que el recipiente fuera empleado para sostener el fuego portátil a manera de braseros o sahumerios similares a los empleados por la sociedad Tiahuanaco (Berenguer, 2000). Los sahumadores Tiahuanaco fueron muy típicos y parte de la parafernalia alfarera junto con los keros y huaco-retratos; sin embargo en la sociedad Moche se desconoce o se ha investigado poco para saber si las actividades rituales estuvieron acompañadas de incensarios o sahumerios que le den un mayor realce.

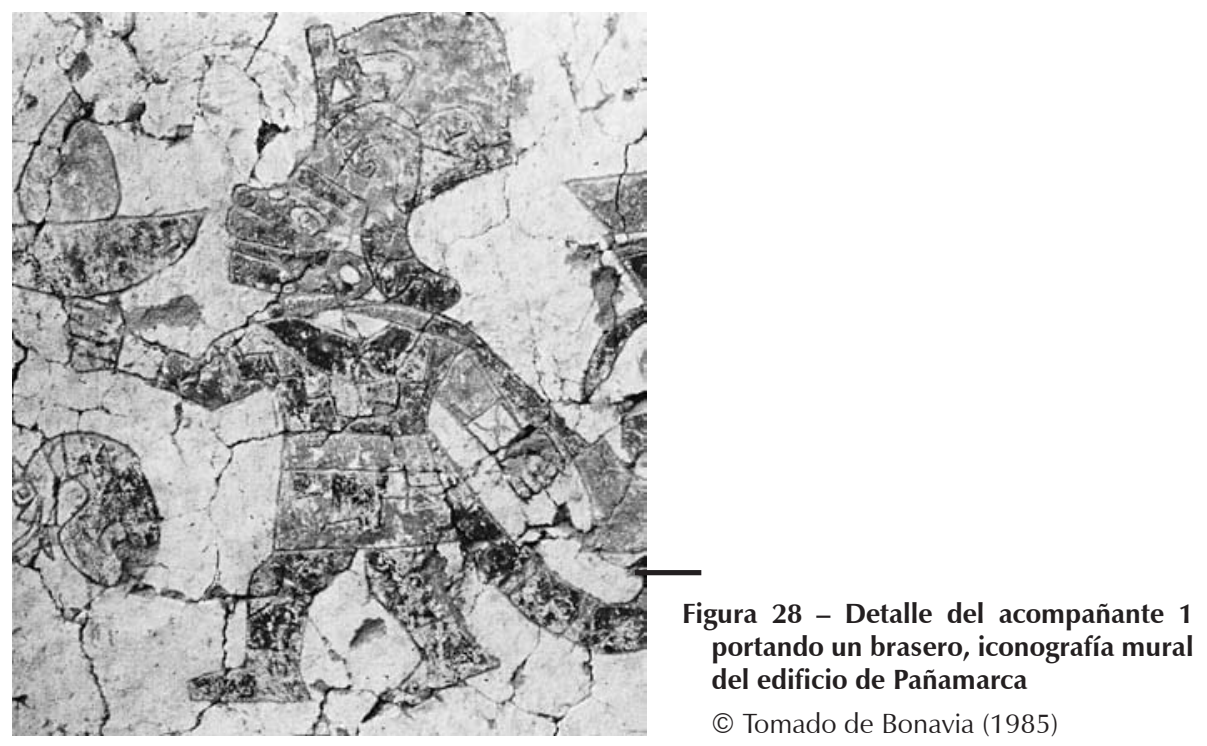


La etnohistoria presenta detalles sobre incensarios empleados en ceremonias rituales de México (Acosta, 1590). El cronista menciona que los sacerdotes portaban un incensario en la mano lleno de brasa, el cual tomaba del brasero o fogón que ardía ante el altar, mientras en la otra mano llevaba una bolsa de incienso, con el cual incensaban a los ídolos con mucha recurrencia. Si bien es cierto, Acosta brinda detalles de la forma cómo eran utilizadas estas vasijas, no queda claro cuál fue el tipo o formas de estas.

La portabilidad de estos objetos sugiere que debió ser un recipiente pequeño de pasta fina, cuya base pedestal fue propicia para sostenerla con una de las dos manos, mientras la otra mano quedaba libre para abastecer de pequeños carbones y/o incienso. El análisis en microscópico de la parte interior de los cuencos con hollín deberá corroborar la hipótesis; así como el cuidadoso registro de nuevas formas registradas en investigaciones posteriores.

Posiblemente debieron existir otros medios de iluminación fija o portátil; estos podrían ser antorchas o lámparas encendidas con una mecha empapada en vegetales, grasas animales o aceites vegetales que no se han conservado hasta nuestros días (Lieberherr, 2006).

\section{CONCLUSIONES}

La variedad de formas de estructuras de combustión registradas en los diferentes componentes del complejo arqueológico huacas del Sol y de la Luna, nos ha permitido proponer un primer ensayo de tipología, estableciendo tres tipos básicos: los fogones, hornos y braseros. Siendo los fogones el más dominante, representado por el subtipo fosa. A estos tipos de estructuras de combustión debemos agregar dos categorías más, una son las áreas de desechos, a las que hemos denominado concentraciones de ceniza y, la segunda, son los testigos de incendio en arquitectura monumental.

A partir del análisis contextual de las estructuras de combustión y los espacios arquitectónicos asociados, así como los estudios etnohistóricos y etnográficos de las comunidades campesinas de Moche y Virú, hemos propuesto una primera aproximación a las funciones de estos diversos tipos de estructuras de combustión. De este modo, hemos propuesto que los fogones tipo fosas han servido para la cocción de alimentos y producción de chicha; los hornos relacionados a la cocción de cerámica y un caso especial de horno con chimenea que habría funcionado para la fusión de metales; además de pequeños hornos de recocido. Asimismo debieron existir formas de fuego portátil como los braseros relacionados con ceremonias rituales. Esta primera aproximación deberá ser confirmada o modificada con estudios sobre los procesos de formación y transformación de estas estructuras mediante análisis físicoquímicos (CG_MS - CG_C_IRMS, difraccion de rayos X, espectrometría Mossbaüer, ICPMS) (March, 2013; March et al., 2014). Estos deberán estar orientados a la caracterización de los elementos orgánicos e inorgánicos presentes, así como a 
definir las fases minerales, su estado de alteración y las temperaturas alcanzadas durante el funcionamiento de los fogones, estudios antracológicos (para definir la naturaleza de los combustibles y su modo de utilización) y micromorfológicos (Guffroy et al., 1994); así como ensayos experimentales que nos permitan definir su modo de funcionamiento y su función de una manera más precisa. Además de entender la duración de sus respectivas utilizaciones (March et al., 2010; 2014). Estudios que esperamos realizar en colaboración con la Universidad de Rennes 1 y el UMR 6566 del CNRS.

\section{Referencias citadas}

ACOSTA, J. de, 1590 - Historia natvral y moral de las Indias, en las que se tratan las cosas notables del cielo, y elementos, metales, plantas, y animales dellas; y los ritos, y ceremonias, leyes, y gouierno, y gueras de los Indios, 535 pp.; Sevilla: Casa de Juan de León.

ARMAS, J., HUANCA, J., \& SÁNCHEZ, R., 2007 - El conjunto arquitectónico 9: caracterización de la ocupación. In: Investigaciones en la Huaca de la Luna 2001. (S. Uceda, E. Mujica \& R. Morales, eds.): 195-204; Trujillo: Facultad de Ciencias Sociales de la Universidad Nacional de Trujillo.

ARTEAGA, I., ALLUÉ, E., PASTÓ, I., VALLVERDú, J., \& CARBONELL, E., 2001 - Els fogars del Peleolitic Mitjà de Lábric Romaní (Capellades, Anoia). Cypsela, 13: 13-29.

BAR-YOSEF, O., CAVALLI-SFORZA, L., MARCH, R. J., \& PIPERNO, M., 1996 - Colloquia 5. The Lower and Middle Paleolithic, Colloquium IX: The study of human behavior in relation to fire in archaeology: new data and methodologies for understanding prehistoric fire structures et Colloquial X The origin of modern man XIII International Congress of prehistoric and protohistoric sciences, 8/14 September 1996, 272 pp.; Forli: M. Editors.

BERENGUER, J., 2000 - Tiwanaku. Señores del lago sagrado, 107 pp.; Santiago de Chile: Museo Chileno de Arte Precolombino \& Banco Santiago.

BENZONI, J., 1968 - La historia del nuevo mundo, 77 pp.; Lima: Universidad Nacional Mayor de San Marcos.

BONAVIA, D., 1961 - A mochica painting at Panamarca, Peru. American Antiquity, 26 (4): 540-543.

BONAVIA, D., 1985 - Mural painting in ancient Peru, xi+224 pp.; Bloomington: Indiana University Press.

BOURDIEU, P., 1980 - Le sens pratique, 475 pp.; París: Editions de Minuit.

CASTILLO, F., 2012 - Estructuras de combustión. Tipología e implicancias en la modelización del espacio del Núcleo Urbano Moche, 327 pp.; Trujillo: Facultad de Ciencias Sociales de la Universidad Nacional de Trujillo. Tesis de Licenciatura.

CHAPDELAINE, C., 1998 - Excavaciones en la zona urbana de Moche durante 1996. In: Investigaciones en la Huaca de la Luna 1996 (S. Uceda, E. Mujica \& R. Morales, eds.): 85-115; Trujillo: Facultad de Ciencias Sociales de la Universidad Nacional de la Libertad.

CHÁVARRI, H. \& MEJÍA, J., 2011 - Excavaciones arqueológicas en Plaza 2a - temporada 2010. In: Proyecto Arqueológico Huaca de la Luna, Informe técnico 2010 (S. Uceda \& 
R. Morales, eds.): 125-146; Trujillo: Facultad de Ciencias Sociales de la Universidad Nacional de Trujillo.

CHIGUALA, J., GAMARRA, N., GAYOSO, H., PRIETO, G., RENGIFO, C. \& ROJAS, C., 2012 - Dinámica ocupacional del Conjunto Arquitectónico 27. Núcleo urbano del Complejo Arqueológico Huacas del Sol y La Luna. In: Investigaciones en la Huaca de la Luna 2003 (S. Uceda, E. Mujica \& R. Morales, eds.): 101-169; Trujillo: Facultad de Ciencias Sociales de la Universidad Nacional de Trujillo.

CHIGUALA, J., RODRÍGUEZ, F., MORALES, A., SOTO, P., LÓPEZ, M. \& GÓMEZ, J., 2007 Áreas de actividad del Conjunto Arquitectónico 21 y su integración al Bloque Constructivo N. ${ }^{\circ}$ 2. In: Proyecto Arqueológico Huaca de la Luna, Informe técnico 2006 (S. Uceda \& R. Morales, eds.): 89-155; Trujillo: Facultad de Ciencias Sociales de la Universidad Nacional de Trujillo.

DE LA VEGA, I. G., 1991 - Los comentarios reales de los Incas, 2 t., 880 pp.; Lima: Fondo de Cultura Económica.

DONNAN, C., 1975 - The thematic approach to Moche iconography. Journal of Latin American Lore, 1 (2): 147-162; Los Ángeles: Latin American Center, University of California.

DONNAN, C., 1998 - Un ceramio moche y la fundición prehispánica de metales. Boletín del Museo Chileno de Arte Precolombino, n..$^{\circ}$ 7: 9-18.

FRANCO, R. \& VILELA, J., 2003 - Aproximaciones al calendario ceremonial mochica del complejo el brujo, valle Chicama. In: Moche: hacia el final del milenio (S. Uceda \& E. Mujica, eds.): 383-423; Lima: Universidad Nacional de Trujillo, Pontificia Universidad Católica del Perú. Actas del Segundo Coloquio sobre la Cultura Moche (Trujillo, 1 al 7 de agosto de 1999).

FRARESSO, C., 2007 - L'usage du métal dans la parure et les rites de la culture Mochica (150-850 AP J-C.), Pérou, 596 pp.; Bordeos: Université Michel de Montaigne de Bordeaux 3. Tesis doctoral.

FRARESSO, C., 2010 - Estudio arqueometalúrgico de un taller de transformación de cobre y de aleaciones tumbaga en el sitio de huacas de Moche. Bulletin de I'Institut Français d'Études Andines, 39 (2): 351-387.

FRÈRE-SAUTOT, M-C., VICHERD, G., VILLES, A., BONTEMPS, C. \& CHASTEL, J., 2003 Le Feu domestique et ses structures au Néolithique et aux âges des métaux: Actes du colloque de Bourg-en-Bresse et Beaune, 7 et 8 octobre 2000, 560 pp.; Montagnac: M. Mergoil.

GAYOSO, H., 2010 - Excavaciones en los CA 39 y 43 o Bloque Arquitectónico 4: un espacio de fiesta en El núcleo Urbano de Huacas del Sol y de la Luna. In: Proyecto Arqueológico Huaca de la Luna. Informe Técnico 2009 (S. Uceda \& R. Morales, eds.): 397-432; Trujillo: Facultad de Ciencias Sociales de la Universidad Nacional de Trujillo.

GOUDSBLOM, J., 1992 - Fire and Civilisation, 247 pp.; London: Ed. Allen Lane The Penguin Press.

GUFFROY, J., MARCH, R., \& WATTEZ, J., 1994 - Les structures et produits de combustion. In: Cerro Nañañique: Un établissement monumental de la période formative, en limite de désert (Haut Piura, Pérou) (J. Guffroy, ed.): 137-166; París: Ed. ORSTOM.

LEROI-GOURHAN, A., 1964 - Le geste et la parole vol. I: Technique et Langage, 323 pp.; París: Ed. Albin Michel.

LEROI-GOURHAN, A. \& BRÉZILLON, M., 1972 - Fouilles de Pincevent, essai d'analyse ethnographique d'un habitat magdalénien, 2 vols., 331 pp.; París: CNRS 2. 
LEVI-STRAUSS, C., 1964 - Le cru et le cuit, 402 pp.; París: Ed. Plon.

LIEBERHERR, R., 2006 - Le feu domestiqué, usage et pratiques dans I'architecture mondiale, 54. Établissement Humains et Environnement Socio-Culturel. Propuesta de Aubry para el estudio de la arquitectura vernacular, 156 pp.; París: Unesco.

MAKOWSKI, K. \& VELARDE, M. I. 1998 - Taller de Yécala (siglo III/IV): observaciones sobre las características y organización de la producción metalúrgica Vicús. Boletín Museo de Oro, vol. 41: 99-118.

MARCH, R. J., 2002 - II controllo del fuoco, in II Mondo dell, Archeologia, vol. II. Enciclopedia Italiana Treccani: 807-812.

MARCH, R. J., 2013 - Searching for the Functions of Fire Structures in Eynan (Mallaha) and their Formation Processes: a Geochemical Approach. In: Natufian Foragers in the Levant: Terminal Pleistocene Social Changes in Western Asia (O. Bar-Yosef \& F. Raymond, eds.): 227-284; Ann Arbor, Michigan. Archaeological Series 19 International Monographs in Prehistory.

MARCH, R. J. \& WÜNSCH, G., 2003 - Loupes et lentilles obscures: à propos de la fonction des structures de combustion. In : Le feu domestique et ses structures au néolithique et aux âges des métaux (M. Mergoil, ed.): 311-318. Actes du colloque Bourg-enbresse 7 octobre 2000, Beaune 8 octobre 2000.

MARCH, R. J., BALDESSARI, A. \& GROSS, E., 1989 - Determinación de compuestos orgánicos en estructuras de combustión arqueológicas. Memoires du Musée de Préhistoire D'lle de France, 2: 47-58.

MARCH, R. J., MUHIEDDINE, M. \& CANOT, E., 2010 - Simulation 3D des structures de combustion préhistoriques. Archéovision, 4: 29-39. Actes du colloque virtual retrospect 2009 Session 1.

MARCH, R. J., LUCQUIN, A., JOLY, D., FERRERI, J. C. \& MUHIEDDINE, M., 2014 Processes of formation and alteration of archaeological fire structures: views on complexity based on experimental approaches. Journal of archaeological method and theory, volumen 21, issue 1, March 2014: 1-47.

MENESES, J., CASTILlO, F., FIGUEROA, M., GARCíA, M., GÓMEZ, B., TORRES, J., VELÁSQUEZ, V. \& VILLANUEVA, L., 2011 - El Bloque Arquitectónico 4: una residencia de élite durante el moche tardío del complejo arqueológico Huacas del Sol y de la Luna. In: Proyecto Arqueológico Huaca de la Luna. Informe Técnico 2010 (S. Uceda \& R. Morales, eds.): 179-283; Trujillo: Facultad de Ciencias Sociales de la Universidad Nacional de Trujillo.

MENESES, J., CASTILLO, F., TORRES, L., RÍOS, J., LA ROSA, V., SANTISTEBAN, S. \& ZÚÑIGA, V., 2012 - Conjunto Arquitectónico 5: Definiendo una nueva residencia en la parte sur del Núcleo Urbano Moche. In: Proyecto Arqueológico Huaca de la Luna. Informe Técnico 2011 (S. Uceda \& R. Morales, eds.): 309-392; Trujillo: Facultad de Ciencias Sociales de la Universidad Nacional de Trujillo.

OLIVE, M. \& TABORIN, Y., 1989 - Nature et fonction des foyers préhistoriques. Actes du colloque international de Nemours, 12-13-14 mai 1987, 334 pp.; Nemours: Association pour la promotion de la recherche archéologique en lle de France.

ORBEGOSO, M., GÓMEZ, J., CHUMBE, L., RAMÍREZ, L. \& BARBOZA, S., 2011 Excavaciones en el Frontis Norte y en la Plaza 1 de Huaca de la Luna. In: Proyecto Arqueológico Huaca de la Luna, Informe técnico 2010 (S. Uceda \& R. Morales, eds.): 51-124; Trujillo: Facultad de Ciencias Sociales de la Universidad Nacional de Trujillo. 
QUILTER, K., 2008 - Art and Moche Martial Arts. In: Moche Art and Archaeology in Ancient Peru (S. Bourget \& K. Jones, eds.): 215-228; Austin: University of Texas Press.

SEOANE, F., CAMPAÑA, V., CASTILLO, F., CHUMBE, L., MEJÍA, J. \& GAMBOA, P., 2010 El Conjunto Arquitectónico N. ${ }^{\circ} 42$ y los bloques arquitectónicos en el Núcleo Urbano Moche. In: Proyecto Arqueológico Huaca de la Luna. Informe Técnico 2009 (S. Uceda \& R. Morales, eds.): 305-395; Trujillo: Facultad de Ciencias Sociales de la Universidad Nacional de Trujillo.

SHIMADA, I., 1994a - Pampa Grande and the Mochica Culture, 341 pp.; Austin: University of Texas Press.

SHIMADA, I., 1994b - La producción de cerámica en Mórrope, Perú: Productividad, especialización y espacio vistos como recursos. In: Tecnología y organización de la producción de cerámica prehispánica en los andes (I. Shimada, ed.): 295-320; Lima: Pontificia Universidad Católica del Perú.

SOLER, B., 2003 - Estudio de las estructuras de combustión prehistóricas: una propuesta experimental. Cova Negra (Xàtiva, Valencia), Ratlla del Bubo (Crevillent, Alicante) y Marolles-sur-Seines (Bassin Parísien, Francia); xii+163 pp.; Valencia: Servicio de Investigación Prehistórica.

TUFINIO, M., 2005 - Excavaciones en el Frontis Norte y Plaza 1 de Huaca de la Luna. In: Proyecto Arqueológico Huaca de la Luna, Informe técnico 2004 (S. Uceda \& R. Morales, eds.): 57-92; Trujillo: Facultad de Ciencias Sociales de la Universidad Nacional de Trujillo.

UCEDA, S., 2010 - Los contextos urbanos de producción artesanal en el complejo arqueológico de las huacas del Sol y de la Luna. Bulletin de I'Institut Français d'Etudes Andines, 39 (2): 243-297.

UCEDA, S. \& ARMAS, J., 1997 - Los talleres alfareros en el centro urbano Moche. In: Investigaciones en la Huaca de la Luna 1995 (S. Uceda, E. Mujica \& R. Morales, eds.): 93-103; Trujillo: Facultad de Ciencias Sociales de la Universidad Nacional de la Libertad.

WRANGHAM, R. W., 2009 - Catching Fire How cooking made us human, 320 pp.; London: Profile Books.

WRIGHT, L., 1966 - Los fuegos del hogar. De la hoguera prehistórica a la cocina y a la calefacción de hoy, 237 pp.; Barcelona: Ed. Noguer S. A.

ZAVALETA, E., ZAPATA, C., AFÁ, G., AVILENO, D., MONTALVO, J., ROBLES, A., ROMÁN, C., ZAGASTIZÁBAL, D., 2011 - Arquitectura y actividades productivas en el CA 44 durante el moche tardío en el Complejo Arqueológico Huacas del Sol y la Luna, Valle de Moche. In: Proyecto Arqueológico Huaca de la Luna. Informe Técnico 2010 (S. Uceda \& R. Morales, eds.): 285-371; Trujillo: Facultad de Ciencias Sociales de la Universidad Nacional de Trujillo. 